\title{
Crystal structure of the TSH receptor (TSHR) bound to a blocking-type TSHR autoantibody
}

\author{
Paul Sanders, Stuart Young, Jane Sanders, Katarzyna Kabelis, Stuart Baker, \\ Andrew Sullivan, Michele Evans, Jill Clark, Jane Wilmot, Xiaoling Hu, Emma Roberts, \\ Michael Powell, Ricardo Núñez Miguel, Jadwiga Furmaniak and Bernard Rees Smith
}

FIRS Laboratories, RSR Ltd, Parc Ty Glas, Llanishen, Cardiff CF14 5DU, UK

(Correspondence should be addressed to B Rees Smith; Email: firs@ rsrltd.eclipse.co.uk)

\begin{abstract}
A complex of the TSH receptor extracellular domain (amino acids 22-260; TSHR260) bound to a blocking-type human monoclonal autoantibody (K1-70) was purified, crystallised and the structure solved at 1.9 $\AA$ resolution. K1-70 Fab binds to the concave surface of the TSHR leucine-rich domain (LRD) forming a large interface $\left(2565 \AA^{2}\right)$ with an extensive network of ionic, polar and hydrophobic interactions. Mutation of TSHR or K1-70 residues showing strong interactions in the solved structure influenced the activity of $\mathrm{K} 1-70$, indicating that the binding detail observed in the complex reflects interactions of K1-70 with intact, functionally active TSHR. Unbound K1-70 Fab was prepared and crystallised to $2 \cdot 22 \AA$ resolution. Virtually no movement was observed in the atoms of $\mathrm{K} 1-70$ residues on the binding interface compared with unbound K1-70, consistent with 'lock and key' binding. The binding arrangements in the TSHR260-K1-70 Fab complex are similar to previously observed for the TSHR260-M22 Fab complex; however, K1-70 clasps the concave surface of the TSHR LRD in approximately the opposite orientation (rotated $155^{\circ}$ ) to $\mathrm{M} 22$. The blocking autoantibody $\mathrm{K} 1-70$ binds more N-terminally on the TSHR concave surface than either the stimulating autoantibody M22 or the hormone TSH, and this may reflect its different functional activity. The structure of TSHR260 in the TSHR260-K1-70 and TSHR260-M22 complexes show a root mean square deviation on all $\mathrm{C}_{\alpha}$ atoms of only $0.51 \AA$. These high-resolution crystal structures provide a foundation for developing new strategies to understand and control TSHR activation and the autoimmune response to the TSHR.
\end{abstract}

Journal of Molecular Endocrinology (2011) 46, 81-99

\section{Introduction}

The TSH receptor (TSHR) is a major autoantigen in autoimmune thyroid disease, and TSHR autoantibodies with thyroid-stimulating (agonist) activity are responsible for the hyperthyroidism of Graves' disease (Rees Smith et al. 1988, 2007, Rapoport et al. 1998, Sanders et al. 1997). However, in rare cases, TSHR autoantibodies act as antagonists and prevent the TSHR-binding and stimulating activities of TSH and can cause hypothyroidism (Rees Smith et al. 1988, 2007, McKenzie \& Zakarija 1992, Sanders et al. 1997, Rapoport et al. 1998). The availability of human monoclonal antibodies to the TSHR with the characteristics of patient serum autoantibodies has allowed major developments in our understanding of the TSHR-autoantibody interaction, and in the case of thyroid-stimulating autoantibodies, the crystal structure of a human monoclonal autoantibody (M22) in complex with the TSHR (amino acids 22-260; TSHR260) has been determined at 2.55 Å resolution (Sanders et al. 2007a).

Recently, we have isolated two new human monoclonal autoantibodies to the TSHR from a single sample of a patient's peripheral blood lymphocytes
(Rees Smith et al. 2009, Evans et al. 2010). One monoclonal antibody is a powerful thyroid stimulator (K1-18), and the other (K1-70) blocks the stimulating activities of TSH and TSHR autoantibodies (including M22 and K1-18). We now describe the crystal structure of K1-70 Fab unbound (solved at 2.22 $\AA$ ) and the crystal structure of K1-70 Fab bound to TSHR260 (solved at $1.9 \AA$ ). Using the information obtained from the crystal structures, we then carried out a molecular level analysis of the antigen-binding surfaces of K1-70 (antagonist) and M22 (agonist) Fabs as well as a detailed comparison of the interactions of K1-70 and M22 with the TSHR.

\section{Materials and methods}

\section{Preparation of K1-70 Fab for crystallisation}

K1-70 IgG was prepared from heterohybridoma culture supernatants using protein A affinity chromatography on MabSelect (GE Healthcare, Little Chalfont, UK) according to the manufacturer's instructions. The purified IgG was treated with mercuripapain 
(Sigma-Aldrich, Poole, UK) at an enzyme/protein $(\mathrm{w} / \mathrm{w})$ ratio of 1:200, EDTA at a final concentration of $2 \mathrm{mmol} / \mathrm{l}$ and L-cysteine at a final concentration of $1 \mathrm{mmol} / \mathrm{l}$ for $1 \mathrm{~h}$ at $37^{\circ} \mathrm{C}$. The reaction was terminated by the addition of $50 \mathrm{mmol} / \mathrm{l}$ iodoacetamide in $2 \mathrm{~mol} / \mathrm{l}$ Tris- $\mathrm{HCl}(\mathrm{pH} 8 \cdot 3)$ for $30 \mathrm{~min}$ at room temperature. The digest was passed through a MabSelect column to separate any intact IgG or Fc from the Fab preparation. K1-70 Fab for crystallisation was concentrated to $15.5 \mathrm{mg} / \mathrm{ml}$ using iCON concentrators $(20 \mathrm{ml}$, 9K MWCO; ThermoFisher Scientific, Cramlington, UK). The integrity of the K1-70 Fab was analysed by SDS-PAGE, size-exclusion HPLC on a TSK gel G3000 SW column (Tosoh, Sigma-Aldrich), and the ability of K1-70 Fab to inhibit TSH-biotin binding to the TSHR was tested as described previously (RSR Ltd, Cardiff, UK; Bolton et al. 1999).

\section{$\mathrm{X}$-ray diffraction and structure determination of K1-70 Fab}

K1-70 Fab crystals were grown using the hanging drop vapour diffusion method with the structure screen 1 sparse matrix screen from Molecular Dimensions Ltd (Soham, UK). Condition 24 (30\% PEG 400, 0·1 M sodium HEPES, 0.2 M magnesium chloride, $\mathrm{pH} 7 \cdot 5$ ) yielded single crystals suitable for X-ray diffraction data collection. A single crystal of dimensions $0.3 \times 0.2$ $\times 0.2 \mathrm{~mm}$ was flash cooled in liquid nitrogen. X-ray diffraction data collections were performed at cryogenic temperatures. The dataset was collected using a Rigaku-007HF generator and an R-Axis IV image plate detector, and was indexed, integrated and scaled using MOSFLM and SCALA (Biofocus, Saffron Walden, UK).

The structure of K1-70 Fab was determined by the molecular replacement method using the pdb coordinates of 1LIL ( $\mathrm{V}_{\mathrm{L}}$ and $\mathrm{C}_{\mathrm{L}}$ domains), 2BOS ( $\mathrm{V}_{\mathrm{H}}$ domain) and 2EH7 ( $\mathrm{V}_{\mathrm{L}}$ domain). Molecular replacement was performed using the program PHASER, and the resulting model given ten cycles of atomic refinement with tight geometric weights using REFMAC5 (Collaborative Computational Project number 4[CCP4] 1994). Electron density maps calculated after initial refinement were examined using COOT (Emsley et al. 2010) and automatically corrected and rebuilt using BUCCANEER (CCP4 1994). The structural geometry of K1-70 Fab was checked using PROCHECK (Laskowski et al. 1993, CCP4 1994) and RAMPAGE (CCP4 1994). Electrostatic potential surfaces of the antigen-binding sites were generated using PYMOL (DeLano 2002).

\section{Preparation of the TSHR260-K1-70 Fab complex}

A TSHR260 construct (coding for amino acids (aa) 1-260 of the human TSHR [764aas]) was produced and expressed in insect cells using the baculovirus system as described previously (Sanders et al. 2007a). Purified K1-70 Fab was added to High Five insect cell cultures $96 \mathrm{~h}$ post infection to a final concentration of $2 \mu \mathrm{g} / \mathrm{ml}$. Culture supernatants containing TSHR260K1-70 Fab complex were harvested $120 \mathrm{~h}$ post infection by centrifugation at $500 \mathrm{~g}$ for $10 \mathrm{~min}$. One tablet of complete protease inhibitors (Roche Diagnostics, Lewes, UK) was added per $200 \mathrm{ml}$ supernatant, before storing at $-70{ }^{\circ} \mathrm{C}$ until purification.

\section{Purification of TSHR260-K1-70 Fab complex}

Culture supernatant containing the TSHR260-K1-70 Fab complex was adjusted to $\mathrm{pH} 6.4$ with $500 \mathrm{mmol} / 1$ $\mathrm{NaH}_{2} \mathrm{PO}_{4}$ and loaded onto $75 \mathrm{ml}$ Streamline Direct HST-1 matrix in a Streamline 25 expanded bed chromatography system (GE Healthcare), washed in $100 \mathrm{mmol} / \mathrm{l} \mathrm{NaCl}$ and $50 \mathrm{mmol} / \mathrm{l}$ Tris-HCl (buffer A) $\mathrm{pH} 6 \cdot 0$, and then in buffer $\mathrm{A} \mathrm{pH} 6.5$ and eluted in buffer A $\mathrm{pH} 8 \cdot 5$. The presence of the TSHR260-K1-70 Fab complex in the eluted fractions was confirmed by western blot analysis using a mouse monoclonal antibody $(1 \mu \mathrm{g} / \mathrm{ml})$ reactive with a TSHR epitope within amino acids 246-260 (TSHR MAb 18C5; Jeffreys et al. 2002).

The TSHR260-K1-70 Fab complex was further purified by affinity chromatography using a mouse monoclonal antibody (TSHR MAb 14C4; Jeffreys et al. 2002) that binds to a conformational epitope within amino acids 22-261 of the TSHR extracellular domain coupled to CNBr-activated sepharose-4B (SigmaAldrich). The dialysed complex was then further purified using nickel affinity chromatography on an Ni-NTA agarose column (Qiagen, Crawley, UK). The complex was eluted with $80 \mathrm{mmol} / \mathrm{l}$ imidazole, $100 \mathrm{mmol} / 1 \mathrm{NaCl}$ and $50 \mathrm{mmol} / 1$ Tris-HCl $(\mathrm{pH} 8 \cdot 0)$, dialysed into $100 \mathrm{mmol} / \mathrm{l} \mathrm{NaCl}, 50 \mathrm{mmol} / \mathrm{l}$ Tris-HCl $(\mathrm{pH} 8 \cdot 0)$ and used to set up deglycosylation reactions.

\section{Deglycosylation of the TSHR260-K1-70 Fab complex}

The purified complex was deglycosylated using Endoglycosidase F3 (Sigma-Aldrich) at an enzymeto-complex ratio of $152 \mathrm{mU}$ of enzyme per $\mathrm{mg}$ of complex in $50 \mathrm{mmol} / \mathrm{l}$ sodium acetate buffer $(\mathrm{pH} 4.5)$ at $20^{\circ} \mathrm{C}$ for 5 days. The deglycosylation reactions were then adjusted to $\mathrm{pH} 6.5$ using $200 \mathrm{mmol} / \mathrm{l}$ Tris- $\mathrm{HCl}$, run onto a cation exchange HPLC Bioassist $\mathrm{S}$ column (Tosoh) using an Akta (GE Healthcare) platform. The complex was eluted using a $\mathrm{pH}$ gradient from $6 \cdot 5$ to $9 \cdot 0$.

Eluted complex was further purified by size-exclusion chromatography. TSHR260-K1-70 Fab preparations were applied to a Superdex 200 XK-26 preparative column in $150 \mathrm{mmol} / \mathrm{l} \mathrm{NaCl}$ and $10 \mathrm{mmol} / \mathrm{l}$ Tris-HCl $(\mathrm{pH} 7 \cdot 6)$, and purified complex eluted from the 
column was adjusted to $20 \mathrm{mmol} / \mathrm{l}$ Tris- $\mathrm{HCl}$ and $25 \mathrm{mmol} / \mathrm{l} \mathrm{NaCl}$, pH 7.6. The complex was concentrated using an iCON concentrator $(7 \mathrm{ml} 9 \mathrm{~K}$ MWCO; ThermoFisher) followed by a MicroCon YM-10 concentrator (Millipore, Watford, UK) to a final concentration of $10 \mathrm{mg} / \mathrm{ml}$.

\section{X-ray diffraction and structure determination of TSHR260-K1-70 Fab}

Crystals of TSHR260-K1-70 Fab complex were obtained in 96-well plates using the PEG/Ion HT screen from Hampton Research (Aliso Viejo, CA, USA). The best conditions were optimised further in 24-well plates using a range of PEG 3350 concentration from 16 to $26 \%$, and the best crystals grew in $16 \%$ PEG $3350,0 \cdot 2 \mathrm{M}$ sodium malonate, $\mathrm{pH} 5 \cdot 0$. A single crystal (dimensions $0 \cdot 3 \times 0 \cdot 1$ $\times 0.1 \mathrm{~mm}$ ) was transferred to a solution containing $22 \%$ PEG 3350, $0 \cdot 2 \mathrm{M}$ sodium malonate, $\mathrm{pH} 5 \cdot 0$, and flash frozen in liquid nitrogen. The dataset was collected on a Rigaku-007HF generator and an R-Axis IV image plate detector, and was indexed, integrated and scaled using MOSFILM and SCALA (Biofocus).

The unbound K1-70 Fab crystal structure and the TSHR260 crystal structure (Sanders et al. 2007a) were used for molecular replacement using the programs PHASER and REFMAC5 (CCP4 1994). Structural geometry was checked using PROCHECK and RAMPAGE (CCP4 1994). Electrostatic potential surfaces were generated using PYMOL (DeLano 2002). The TSHR-K1-70 structure has been deposited in the Protein Data Bank with accession number 2XWT.

\section{Preparation of recombinant K1-70 Fab}

The K1-70 heavy-chain (HC) RT-PCR product and the light-chain (LC) RT-PCR product with ' $\mathrm{C}$ ' terminal six histidine tags were cloned into the Immunozap H/L vector (Stratagene Europe, Amsterdam, The Netherlands) into XhoI and SpeI, and SacI and XbaI restriction sites respectively (Sanders et al. 2004). Specific 'forward' and 'reverse' primers were designed to change the K1-70 nucleotide sequence to code for the appropriate amino acid mutation (Sanders et al. $2007 b$ ). Amino acid mutations in the K1-70 Fab HC or LC sequence were introduced using the Qwikchange Mutagenesis Kit (Stratagene) following the manufacturer's instructions. The presence of the wild-type or mutated HC or LC was verified by sequence analysis (Source BioScience, Cambridge, UK).

Plasmid DNA containing the K1-70 HC and LC sequences was transformed into HB2151 cells (GE Healthcare), and expression of recombinant Fab was induced in the presence of $1 \mathrm{mmol} / 1$ isopropyl- $\beta$-D galactoside and $0.3 \mathrm{~mol} / \mathrm{l}$ sucrose. Expression of recombinant Fab was verified using western blot analysis (Birk \& Koepsell 1987) with an anti-human IgG (Fab specific) antibody (SAFC, Poole, UK). Fabs were dialysed into PBS containing $0.2 \mathrm{~g} / \mathrm{l}$ sodium azide and stored at $-70{ }^{\circ} \mathrm{C}$.

Briefly, 41 supernatant containing K1-70 recombinant Fab (K1-70 rFab) were adjusted to $\mathrm{pH} 6.0$ with $500 \mathrm{mmol} / \mathrm{l}$ sodium dihydrogen phosphate $(\mathrm{pH} 4 \cdot 0)$ and loaded onto a $75 \mathrm{ml}$ Streamline TM Direct HST-1 column (GE Healthcare). K1-70 rFab was eluted with $0.3 \mathrm{~mol} / \mathrm{l} \mathrm{NaCl}$ and $10 \mathrm{mmol} / 1 \mathrm{Tris}-\mathrm{HCl}(\mathrm{pH} 8.5)$ and finally purified by chromatography on an Ni-NTA agarose column (Qiagen). The purity of the eluted rFab was $>95 \%$ as assessed by SDS-PAGE (Laemmli 1970), and the concentration of the K1-70 rFab was calculated from the absorbance at $280 \mathrm{~nm}$ on the basis that 1 absorbance unit is equivalent to $0.7 \mathrm{mg} / \mathrm{ml} \mathrm{Fab}$.

Culture supernatants containing non-mutated (wild type; WT) or mutated K1-70 rFab were quantified using the Pierce Easy-Titer Human $\operatorname{IgG}(\mathrm{H}+\mathrm{L})$ assay kit (ThermoFisher) against a standard curve of purified K1-70 rFab as per the manufacturer's instructions.

\section{Inhibition of TSH-biotin binding to the TSHR by wild-type and mutated K1-70 rFabs}

The ability of WT and mutated K1-70 rFabs to inhibit TSH-biotin binding to TSHR-coated ELISA plate wells was determined as described previously (RSR Ltd; Bolton et al. 1999). A calibration curve prepared from purified rK1-70 Fab was included in each assay.

Briefly, $75 \mu \mathrm{l}$ culture supernatant containing either WT or mutated $\mathrm{rFab}$ were diluted in $50 \mathrm{mmol} / 1 \mathrm{NaCl}$, $20 \mathrm{mmol} / 1$ Tris-HCl, $\mathrm{pH} 7 \cdot 8,1 \%$ Triton $\mathrm{X}-100$ and $1 \mathrm{mg} / \mathrm{ml} \mathrm{BSA}$, and after addition of $75 \mu \mathrm{l}$ start buffer incubated in TSHR-coated wells at room temperature for $2 \mathrm{~h}$ with shaking, the assay was carried out as per the kit instructions. Inhibition of TSH-biotin binding was calculated as follows:

$$
100 \times\left(1-\frac{\text { OD } 450 \text { in the presence of test material }}{\text { OD450 in the presence of control material }}\right)
$$

\section{Inhibition of TSH-stimulated cyclic AMP production in CHO cells expressing the TSHR}

The ability of WT K1-70 and mutated K1-70 rFabs to inhibit the production of intracellular cyclic AMP (cAMP) stimulated by porcine (p) TSH was analysed. In these experiments, $\mathrm{CHO}$ cells were seeded into 96-well plates (12 500-20000 cells per well) and incubated for $48 \mathrm{~h}$ in DMEM containing 10\% FCS. The DMEM was removed, and dilutions of WT and mutated K1-70 rFabs $(0 \cdot 001-1 \mu \mathrm{g} / \mathrm{ml}$ final 
concentration) in cAMP assay buffer (NaCl-free Hank's Balanced Salt Solution containing $1 \mathrm{~g} / 1$ glucose, $20 \mathrm{mmol} / 1$ HEPES, $222 \mathrm{mmol} / 1$ sucrose, $15 \mathrm{~g} / 1 \mathrm{BSA}$ and $0.5 \mathrm{mmol} / 1$ 3-isobutyl-1-methylxanthine, $\mathrm{pH} 7 \cdot 4$ ) in the presence of $3 \mathrm{ng} / \mathrm{ml} \mathrm{pTSH}$ were added, and cells were incubated at $37{ }^{\circ} \mathrm{C}$ in an atmosphere of $5 \% \mathrm{CO}_{2}$ in air for $1 \mathrm{~h}$. After removal of test solutions, cells were lysed by the addition of $0.1 \mathrm{~mol} / 1 \mathrm{HCl}, 1 \%$ Triton $\mathrm{X}-100$, and the cAMP concentration in the lysates was determined using the Direct Cyclic AMP Correlate EIA kit (Assay Designs, Inc., Ann Arbor, MI, USA).

In a separate series of experiments, the effect of native K1-70 Fab on pTSH-mediated cAMP production was tested using Flp-In CHO cells expressing mutated TSHR as described previously (Sanders et al. 2006).

\section{Binding of K1-70 to alkaline phosphatase-labelled TSHR260 (TSHR260-AP)}

Briefly, $75 \mu \mathrm{l}$ start buffer (Bolton et al. 1999) and $75 \mu \mathrm{l}$ human monoclonal antibody diluted in a pool of healthy blood donor (HBD) sera were added to ELISA plate wells coated with the full-length detergent solubilised TSHR and incubated for $30 \mathrm{~min}$ at room temperature with shaking (500 shakes per minute). Then the well contents were aspirated, and the wells were washed three times with wash buffer $(50 \mathrm{mmol} / \mathrm{l}$ $\mathrm{NaCl}, 20 \mathrm{mmol} / \mathrm{l}$ Tris, $\mathrm{pH} 7 \cdot 8,1 \%$ Triton X-100) followed by the addition of $100 \mu \mathrm{l}$ TSHR260-AP (diluted in wash buffer containing $0 \cdot 2 \mathrm{~g} / \mathrm{l} \mathrm{MgCl}_{2} \cdot 6 \mathrm{H}_{2} \mathrm{O}$ and $2 \mathrm{~g} / 1 \mathrm{BSA}$; Rees Smith et al. 2009). After incubation for $30 \mathrm{~min}$ at room temperature with shaking (500 shakes per minute), the wells were emptied, washed three times followed by the addition of $100 \mu \mathrm{l}$ p-nitrophenyl phosphate ( $\mathrm{pNpp}$ ) substrate (Europa Bioproducts Ltd, Ely, Cambridge UK), and the plate was incubated in the darkness for $45 \mathrm{~min}$. The reaction was stopped by the addition of $50 \mu \mathrm{l}$ of $1 \mathrm{~mol} / \mathrm{l} \mathrm{NaOH}$, and the absorbance was read at $405 \mathrm{~nm}$.

\section{Results}

\section{Crystal structure of K1-70 Fab}

The structure of K1-70 Fab was determined at $2 \cdot 22 \AA$ resolution with a $R_{\text {work }}$ factor of $21.7 \%$ and a $R_{\text {free }}$ factor of $26.4 \%$ (Supplementary Table 1, see section on supplementary data given at the end of this article). The asymmetric unit contains two complete Fab K1-70 molecules, $\mathrm{Fab}_{\mathrm{A}}$ and $\mathrm{Fab}_{\mathrm{B}}$, that are not related by noncrystallographic symmetry due to differences in elbow angles $\left(\mathrm{Fab}_{\mathrm{A}}=145 \cdot 5^{\circ}\right.$ and $\left.\mathrm{Fab}_{\mathrm{B}}=163 \cdot 1^{\circ}\right)$. The structure of K1-70 is that of a standard Fab fragment with no glycosylation. The distribution of CDRs making up the binding site is standard, with CDRs HC3 and LC3 having a central position, HC1 and LC1 positioned further out, and HC2 and LC2 on the periphery.

Disulphide bonds are present between LC residues 23 and 88, 134 and 194, and between HC residues 22 and 92 and 142 and 208. There are 158 hydrogen bonds within the LC and 177 hydrogen bonds within the HC residues. The accessible surface area (ASA) buried in the interface in the crystal structure is $1717 \AA^{2}$ for the LC and $1592 \AA^{2}$ for the HC residues. The structure of K1-70 lacks electron density of the side chains R94 (CDR3 LC) and R58 (CDR2 HC). After addition of these side chains, the ASAs were recalculated as $1722 \AA^{2}$ for the LC and $1607 \AA^{2}$ for the HC residues. There are 47 residues in the interface, and $40.4 \%$ of these residues are polar; this is similar to M22, which has 43 residues in the interface, and $41.9 \%$ are polar (Sanders et al. 2004).

The combining region of the K1-70 antigen-binding site (Fig. 1) is a highly irregular surface dominated by acidic patches on one side and basic patches on the other side. The acidic patches are centred on D27B (CDR1 LC), D50 (CDR2 LC), D92 (CDR3 LC), D31 (CDR1 HC), D54 and D56 (CDR2 HC) and D96 (CDR3 $\mathrm{HC})$. The basic patches are centred on residues $\mathrm{K} 53$ and R54 (CDR2 LC), R94 (CDR3 LC), R58 (CDR2 HC) and R101 (CDR3 HC), and outside the variable domain region K66 LC creates a basic patch. The surface of K1-70 Fab also contains aromatic residues from both the variable regions and residues located close to them (14 residues in total; Fig. 1). The surface of the combining region contains a cavity at the centre surrounded mostly by aromatic residues and one charged residue D50 LC. Furthermore, the inside of the cavity is lined by aromatic residues.

In comparison, the surface of the thyroid-stimulating human monoclonal autoantibody (M22) antigenbinding site is also highly charged with acidic patches on one side and basic patches on the other side (Fig. 1; Sanders et al. 2004).

\section{Crystal structure of the TSHR260-K1-70 Fab complex}

The structure of the TSHR260-K1-70 Fab complex was solved to $1.9 \AA$ resolution (Fig. 2A), and the crystallographic analysis is summarised in Supplementary Table 2, see section on supplementary data given at the end of this article.

The structure of TSHR260 solved from the TSHR260-K1-70 Fab complex is similar to that previously determined from the TSHR260-M22 Fab complex $(2 \cdot 55 \AA$ resolution; Sanders et al. 2007a). A comparison of these two TSHR260 structures shows a root mean square deviation (rmsd) on all $\mathrm{C}_{\alpha}$ atoms of only $0.51 \AA$, with the highest deviation of $2.42 \AA$ found in the $\mathrm{C}_{\alpha}$ atom of H248. 
As shown in Fig. 2 and described previously (Sanders et al. 2007a), TSHR260 has the shape of a slightly curved helical tube constructed from leucine-rich repeat motifs. It has opposed concave and convex surfaces, with an eleven-stranded $\beta$-sheet located on the concave surface (ten parallel strands, one per repeat and an anti-parallel strand at the N-terminus). The inner surface of the tube is lined with hydrophobic residues. The concave surface of TSHR260 is formed from an untwisted $\beta$-sheet (Fig. 2A), and its convex surface presents eight small strands (two residues each) forming two 3-stranded $\beta$-sheets and one 2-stranded $\beta$-sheet (Fig. 2A). There are five glycosylation sites (N77, N99, N113, N177 and N198) located on the convex surface, and all are glycosylated, although the sugars are too disordered to model with confidence at position N113. The structure of TSHR260 shows a nonbonded cysteine at residue 176 , which is located in the convex surface but with its side chain placed within the hydrophobic core preventing possible disulphide bonding.

The N-terminal cysteines (C31 and C41) in the TSHR sequence are disulphide bonded. The new structure of TSHR260 solved at $1.9 \AA$ shows residues C24, S25, S26, P27, P28 and C29 (Fig. 2B), while there was no electron density for these residues in the TSHR260-M22 Fab complex solved at $2.55 \AA$ (Sanders et al. 2007a).
The new TSHR260 crystal structure provides therefore details of disulphide bonding arrangements at the TSHR N-terminus and shows disulphide bonds between the 1st and 2nd cysteines (C24 and C29) and between the 3rd and 4th cysteines (C31 and C41; Fig. 2B).

\section{Interactions in the TSHR260-K1-70 Fab complex}

K1-70 Fab binds to the concave surface of the TSHR260 and is positioned more N-terminally than the M22 Fab (Sanders et al. 2007a). Also, K1-70 Fab is bound at an approximate rotation of $155^{\circ}$ on the TSHR260 with respect to the position of M22 Fab, and the orientation of the K1-70 and M22 Fab HCs and LCs are opposite (Fig. 3). A comparison of the structure of bound K1-70 Fab in the TSHR260-K1-70 Fab complex and the structure of unbound K1-70 Fab shows that the majority of the residues located on the binding surface of the antibody variable domains have almost identical positions (rmsd of all $\mathrm{C}_{\alpha}$ atoms of the variable domains is $0.45 \AA$; Supplementary Figure 1, see section on supplementary data given at the end of this article). The highest deviation for a backbone $\mathrm{C}_{\alpha}$ atom is $1.29 \AA$ observed for LC G16. In addition, only six of the bound K1-70 Fab residue side chains deviate by more than $3 \AA$ from their positions in unbound K1-70 Fab (LC residues V19, R54 and T80, and HC residues Q3, N76 and R101).
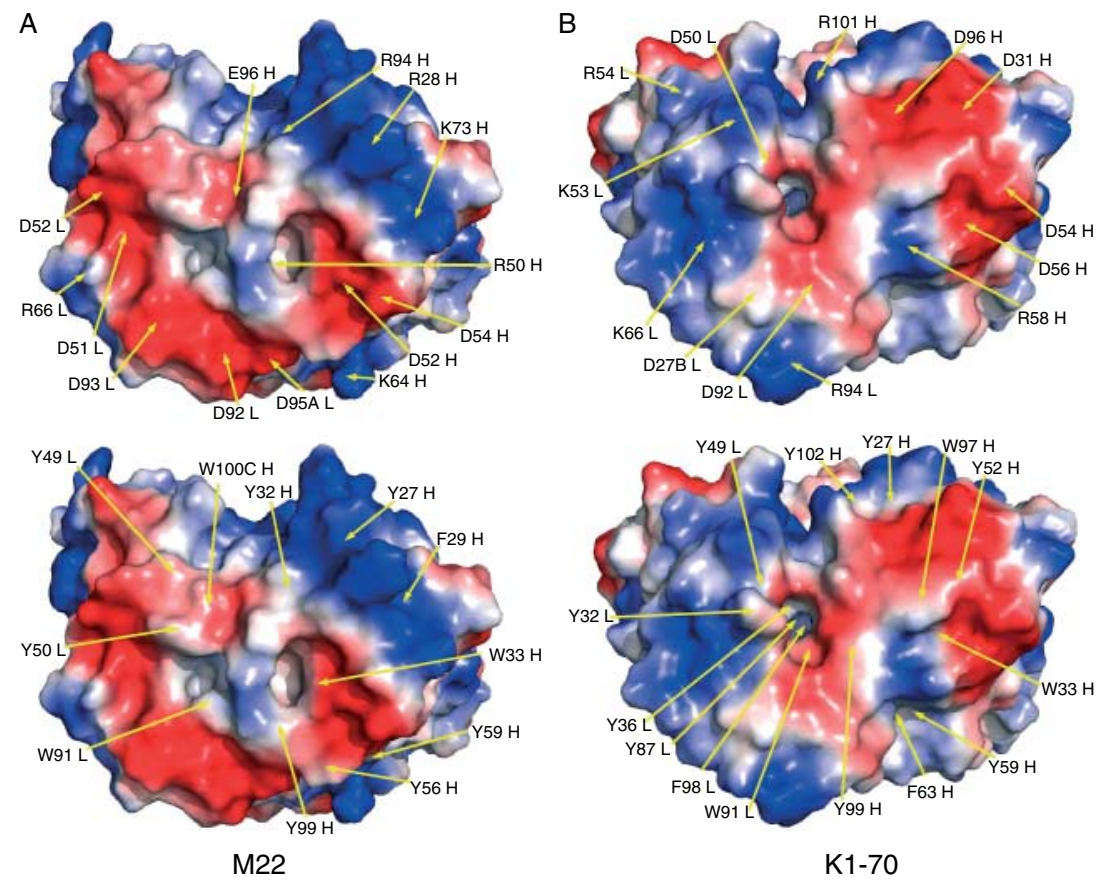

Figure 1 The electrostatic surface potentials (in Pymol; DeLano 2002) of the antigenbinding regions of $(A)$ thyroid-stimulating monoclonal autoantibody (M22) and (B) TSH receptor-blocking monoclonal autoantibody (K1-70) showing the positions of charged residues (top figures) and the positions of aromatic residues (bottom figures). Acidic patches are shown in red, and basic patches are shown in blue. 


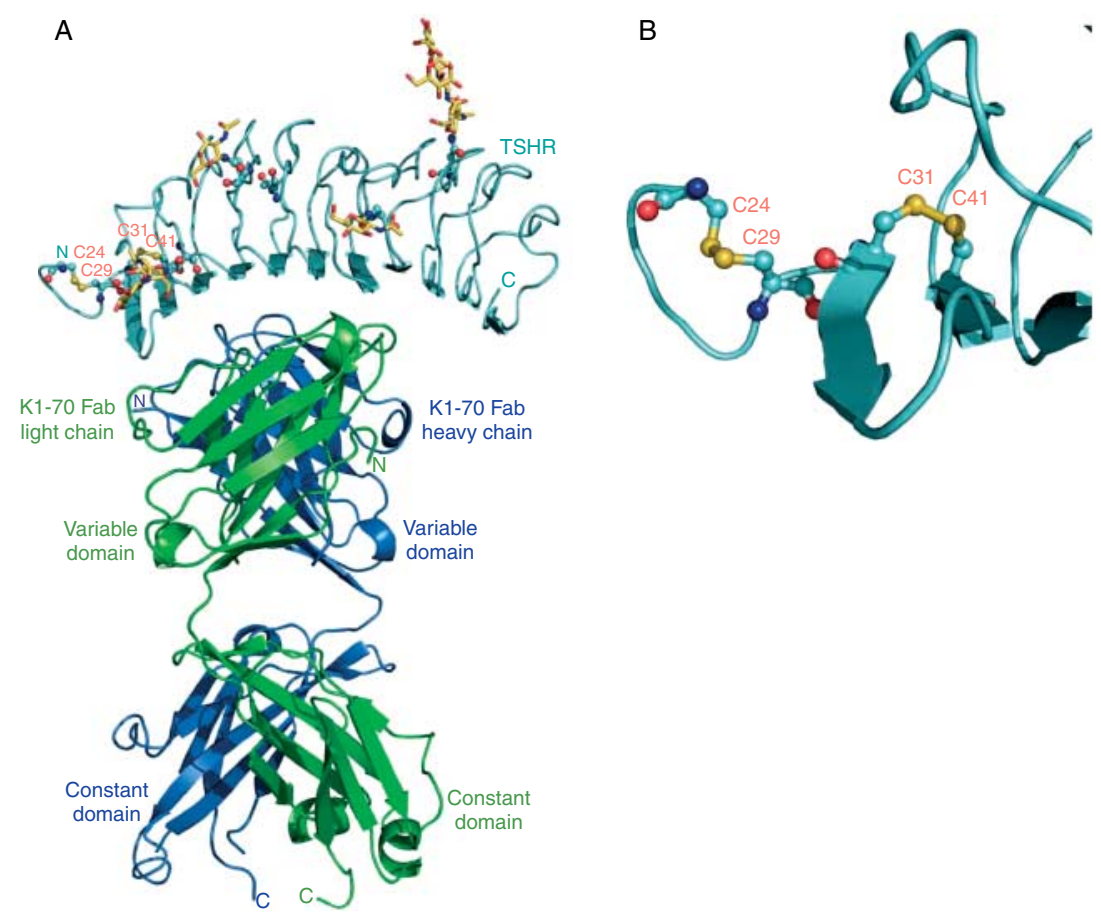

Figure 2 The complex of the TSH receptor leucine-rich repeat domain (TSHR LRD) and the human monoclonal autoantibody $\mathrm{K} 1-70 \mathrm{Fab}$ at $1.9 \AA$ resolution. (A) Cartoon diagram of the complex structure. TSHR is in cyan, K1-70 light chain is in green, and K1-70 heavy chain is in blue. Disulphide-bonded cysteines are shown as ball and stick, disulphide bonds are in yellow, and cysteine residues are labelled in orange. The positions of amino $(\mathrm{N})$ - and carboxy (C)-termini are indicated. (B) Disulphide bond arrangements in the $\mathrm{N}$-terminal cap of the TSHR LRD in more detail. Cysteines are shown as ball and stick, disulphide bonds are shown in yellow, and cysteine residues are labelled in orange.

All of the glycosylation sites on the TSHR are distant from the K1-70 Fab-binding interface (Fig. 2).

A total of $2565 \AA^{2}$ of solvent ASA is buried in the K1-70-TSHR interface, $731 \AA^{2}$ in the interaction with K1-70 HC and $622 \AA^{2}$ in the interaction with the LC.

The interactions between TSHR260 and K1-70 Fab in the complex are a mixture of an extensive hydrogen bonding and salt bridge network ( 25 hydrogen bonds and salt bridges), 19 water-mediated hydrogen bonds, 11 polar interactions and 19 hydrophobic contacts (Table 1 and Fig. 4). There are 24 TSHR260 residues that form direct interactions with K1-70 Fab, and an additional four residues interact through water molecules. Furthermore, there are 12 K1-70 LC residues and $15 \mathrm{HC}$ residues that interact directly with TSHR260 and an additional three LC residues that interact through water molecules. In particular, TSHR $\mathrm{K} 58$ is hydrogen bonded to three $\mathrm{K} 1-70$ residues (D31 $\mathrm{HC}, \mathrm{N} 32 \mathrm{HC}$ and D96 HC), and the K1-70 residue D96 $\mathrm{HC}$ is hydrogen bonded to two TSHR residues (R38 and K58). In addition, some TSHR260 residues are involved in strong van der Waals interactions with K1-70, in particular TSHR R80 with an interaction surface of $101 \cdot 5 \AA^{2}$ and TSHR F153 of $98 \cdot 3 \AA^{2}$ (Table 2). K1-70 residues involved in van der Waals interactions with TSHR260 with large interacting surface areas include residues in both the $\mathrm{HC}$ and $\mathrm{LC}$, for example W97 HC $\left(117 \cdot 3 \AA^{2}\right)$ and R94 LC $\left(97 \cdot 9 \AA^{2}\right.$; Table 2).

The high resolution $(1.9 \AA)$ of the TSHR260-K1-70 Fab complex and the availability of TSHR residues 24-29 in the structure (these were not present in the TSHR260-M22 complex (Sanders et al. 2007a)) provide new details of the structure of the TSHR N-terminus and reveal that the electrostatic surface potential is negatively charged (Fig. 4D). This is different from the assessment of the electrostatic potential distribution in the structure solved at $2.55 \AA$. The difference is due to disorder in this region of the TSHR260-M22 structure, while the TSHR260-K1-70 structure shows more detail.

The electrostatic surface potential of the TSHR260 concave surface and the K1-70 antigen-binding surface is polarised in terms of charge distribution. The surface distribution of charged residues on K1-70 is complementary to that of TSHR260, with the negatively charged surface of the TSHR260 interacting with the positively charged surface of K1-70 and the positively charged surface of TSHR260 interacting with the negatively charged surface of K1-70 (Fig. 4D). 

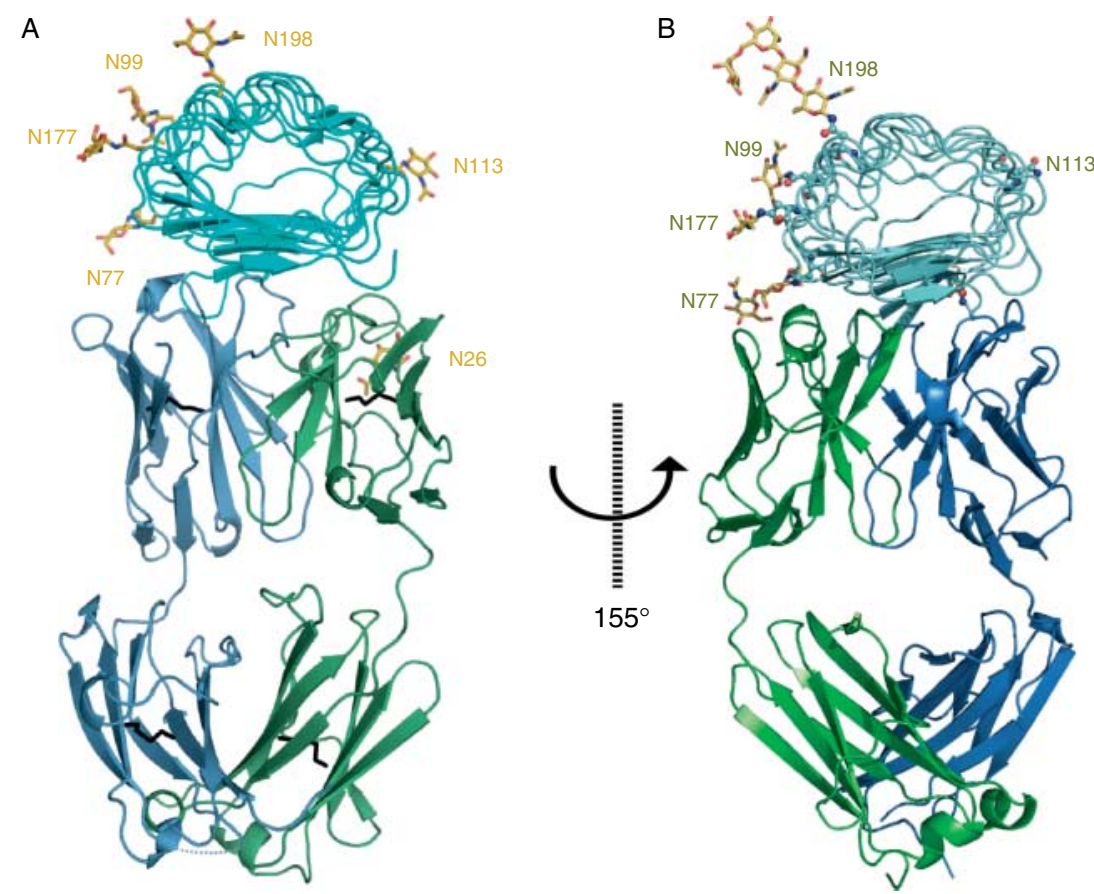

Figure 3 Comparison of the relative positions of the thyroid-stimulating autoantibody M22 and the TSH receptor (TSHR)-blocking monoclonal autoantibody (K1-70) on the TSH receptor leucine-rich repeat domain (TSHR LRD). (A) Crystal structure of TSHR260 in complex with thyroid-stimulating monoclonal autoantibody M22 Fab. TSHR is in cyan, M22 light chain (LC) is in green, and the M22 heavy chain $(\mathrm{HC})$ is in blue. The N-terminal linked carbohydrates observed in the structure are shown in yellow, and carbohydrate bound asparagines residues are labelled. (B) Crystal structure of the TSHR260 in complex with the TSHR-blocking monoclonal autoantibody K1-70 Fab. The TSHR LRD is aligned in the same orientation as the TSHR LRD from the complex with M22 Fab (A). The relative positions of $\mathrm{M} 22$ and $\mathrm{K} 1-70$ Fabs bound to TSHR corresponds to a rotation about the TSHR vertical axis of $\sim 155^{\circ}$.

TSHR D203 is the last residue involved in chargecharge interactions in the TSHR260-K1-70 Fab complex interacting with K1-70 LC R94. The TSHR260 C-terminus is not involved in interactions with the K1-70 Fab (Fig. 2A). There are a number of electrostatic interactions in the TSHR260-K1-70 complex, and these are detailed in Table 3. TSHR R109 produces the strongest accumulated electrostatic interactions with K1-70, whereas K1-70 D31 HC and D50 LC produce the strongest accumulated electrostatic interactions with the TSHR (Table 3).

\section{Effects of mutations in the TSHR and in K1-70 Fab}

We analysed the effects of different amino acid mutations in the TSHR extracellular domain on the ability of K1-70 IgG to block pTSH-induced stimulation of cAMP production in CHO cells expressing the mutated TSHR (Table 4). Mutation of TSHR residues D43, R80, T104, H105, K129, F130, F134, D151, Q235, K250, E251, R255, T257 and W258 to alanine showed no significant effect (compared with wild-type TSHR) on stimulation of cAMP production by TSH or the TSH antagonist activity of K1-70 IgG. Furthermore, K1-70 binds strongly to TSHR260-AP in the bridge ELISA showing a similar dose-dependent response as the thyroid-stimulating monoclonal autoantibody M22. $\mathrm{K} 1-70$ and M22 $\operatorname{IgG}$ at $10 \mathrm{ng} / \mathrm{ml}$ gave an OD of 0.038 and 0.035 respectively (compared with 0.00 for HBD pool only), this increased to 0.993 and 1.045 at $500 \mathrm{ng} / \mathrm{ml}$ and a maximum of 1.416 and 1.532 at $100 \mu \mathrm{g} / \mathrm{ml}$ respectively. However, when full-length TSHR containing the R255D mutation was coated on the ELISA plate wells, K1-70 binding at $100 \mathrm{ng} / \mathrm{ml}$ was not significantly reduced (90\% of WT TSHR binding), while M22 IgG binding was reduced to $13 \%$ of WT TSHR binding.

In the case of TSHR mutations that affected TSH stimulation of cAMP production, i.e. TSHR E107A, E157A, D160A, Y185A, D203A, Y206A, K209A and D232A, no significant effect on the TSH antagonist activity of K1-70 IgG was found. One mutation TSHR E178A caused a slight decrease in activity for both TSH stimulation of cAMP production and inhibition of TSH 
Table 1 Interactions between TSHR260 and K1-70 Fab observed in the crystal structure of the complex

TSHR residue

TSHR atom

$\mathrm{K} 1-70$ residue $^{\mathrm{a}}$

$\mathrm{K} 1-70$ atom

Arg101 HC

Ser56 LC

Asp96 HC

Asp96 HC

Tyr102 HC

Asp31 HC

Lys53 LC

Asp31 HC

Asn32 HC

Asp96 HC

Asp50 LC

Asp50 LC

Asn $100 \mathrm{HC}$

Tyr52 HC

Trp97 HC

Asp54 HC

Asp56 HC

Asp56 HC

Ser30 LC

Arg58 HC

Arg58 HC

Tyr99 HC

Ser93 LC

Arg94 LC

Arg94 LC

Asp203

OD2

OD1

\begin{tabular}{|c|c|}
\hline $\mathrm{NH} 1$ & $2 \cdot 80^{\mathrm{C}}$ \\
\hline $\mathrm{N}$ & 2.99 \\
\hline OD1 & $2 \cdot 74$ \\
\hline OD2 & $2 \cdot 87^{\mathrm{C}}$ \\
\hline $\mathrm{OH}$ & $3 \cdot 25$ \\
\hline OD1 & $2 \cdot 88^{C}$ \\
\hline NZ & $2 \cdot 88$ \\
\hline 0 & $2 \cdot 68$ \\
\hline OD1 & 2.99 \\
\hline 0 & $2 \cdot 78$ \\
\hline OD2 & $2 \cdot 78^{\mathrm{C}}$ \\
\hline OD1 & $2.98^{\mathrm{C}}$ \\
\hline ND2 & 2.92 \\
\hline $\mathrm{OH}$ & $2 \cdot 96$ \\
\hline NE1 & 3.06 \\
\hline OD1 & $2 \cdot 85^{c}$ \\
\hline OD2 & $3.02^{c}$ \\
\hline OD2 & $3 \cdot 23^{c}$ \\
\hline 0 & $2 \cdot 91$ \\
\hline $\mathrm{NH} 1$ & $2 \cdot 81^{\mathrm{c}}$ \\
\hline $\mathrm{NH} 2$ & $3.06^{c}$ \\
\hline $\mathrm{OH}$ & $2 \cdot 79$ \\
\hline 0 & 3.04 \\
\hline NE & 3.03 \\
\hline $\mathrm{NH} 1$ & $2 \cdot 85^{\mathrm{c}}$ \\
\hline
\end{tabular}

\section{TSHR Distance}

TSHR
residue

atom

to water $^{\mathrm{b}}(\AA)$

K1-70 residue ${ }^{a}$

K1-70 atom

Distance

to water ${ }^{\mathrm{b}}(\AA)$

Water

Water-mediated hydrogen bonds

$\begin{array}{lll}\text { Glu35 } & \text { N } & 2 \cdot 97 \\ \text { Asp36 } & \text { O } & 2 \cdot 73 \\ \text { Asp36 } & \text { OD1 } & 2 \cdot 54 \\ \text { Gln55 } & \text { OE1 } & 2 \cdot 80 \\ \text { Thr56 } & \text { OG1 } & 2 \cdot 64 \\ \text { Thr56 } & \text { OG1 } & 2 \cdot 64 \\ \text { Arg80 } & \text { NE } & 2 \cdot 95 \\ \text { Arg80 } & \text { NH2 } & 3 \cdot 19 \\ \text { Lys102 } & \text { O } & 2 \cdot 52 \\ \text { Glu107 } & \text { OE2 } & 2 \cdot 64 \\ \text { Asn110 } & \text { ND2 } & 2 \cdot 66 \\ \text { Lys129 } & \text { NZ } & 3 \cdot 23 \\ \text { Lys129 } & \text { NZ } & 2 \cdot 67 \\ \text { Lys129 } & \text { O } & 2 \cdot 89 \\ \text { Asp151 } & \text { OD1 } & 3 \cdot 05 \\ \text { Phe153 } & \text { O } & 2 \cdot 74 \\ \text { Lys183 } & \text { NZ } & 2 \cdot 56 \\ \text { Tyr206 } & \text { OH } & 2 \cdot 75 \\ \text { Tyr206 } & \text { OH } & 2 \cdot 97\end{array}$

Tyr102 HC

Tyr49 LC

Arg54 LC

Tyr49 LC

Asp96 HC

Asn $100 \mathrm{HC}$

Tyr99 HC

Tyr99 HC

Tyr32 LC

Asn98 HC

Asp54 HC

Gly29 LC

Lys66 LC

Ser93 LC

Lys66 LC

Ser93 LC

Tyr99 HC

Arg94 LC

Arg94 LC w

$3 \cdot 37$

$2 \cdot 80$

3.40

$2 \cdot 80$

$2 \cdot 80$

$2 \cdot 74$

$2 \cdot 85$

$2 \cdot 64$

2.76

2.98

2.91

2.95

$2 \cdot 81$

$2 \cdot 53$

3.30

2.53

$2 \cdot 75$

$2 \cdot 73$

3.20
Distance $(\AA)^{b}$

TSHR

K1-70

TSHR

K1-70

584

218

630

218

94

94

67

693

497

82

124

193

581

191

193

191

343

372

458

Hydrophobic contacts ${ }^{\mathrm{e}}$

Asp36

Arg38

Lys42

Ile60

Pro55 LC

Arg101 HC

Asp31 HC

Asp31 HC

Trp97 HC

(continued) 
Table 1 Continued

\begin{tabular}{|c|c|c|c|}
\hline TSHR & K1-70 & TSHR & K1-70 \\
\hline Arg109 & Arg58 HC & Glu61 & Thr30 HC \\
\hline Asn110 & Asp54 HC & Tyr82 & Trp97 HC \\
\hline Lys129 & Asn31 LC & Ser84 & Trp97 HC \\
\hline \multirow[t]{2}{*}{ Asp151 } & Gly29 LC & Thr104 & Tyr32 LC \\
\hline & Ser30 LC & His105 & Tyr99 HC \\
\hline Phe153 & Ser30 LC & & \\
\hline \multirow[t]{9}{*}{ Lys183 } & Leu95 LC & Glu107 & Trp97 HC \\
\hline & & Arg109 & Trp33 HC \\
\hline & & & Tyr52 HC \\
\hline & & & Trp97 HC \\
\hline & & Phe130 & Tyr99 HC \\
\hline & & Phe153 & Ser27A LC \\
\hline & & & Ser93 LC \\
\hline & & & Arg94 LC \\
\hline & & Ile155 & Ser93 LC \\
\hline
\end{tabular}

aLC, light chain and $\mathrm{HC}$, heavy chain.

${ }^{b}$ Hydrogen bond distances are in the range of $2 \cdot 3-3.4 \AA$.

cDenotes salt bridges.

dPolar contacts have distances between 3.4 and $4.0 \AA$

e Carbon-carbon contacts are within $4 \cdot 0 \AA$.

stimulation by K1-70 $(<100-80 \%)$. In contrast, the TSHR mutations (K58A, I60A, E61A, Y82A, R109A and K183A) showed an absence or a decrease in TSH antagonist activity of K1-70, while TSH stimulation of cAMP production in the mutated TSHR was similar to that in the wild-type TSHR. Analysis of the interactions of these six residues in the TSHR-K1-70 complex showed that all six interacted with K1-70. In particular, K58, Y82 and R109 are involved in 7 out of the 25 hydrogen bonds and salt bridges present in the structure (Table 1), while K183 produces a watermediated hydrogen bond with Y99 of the K1-70 HC. Also R109 and K183 are involved in non-hydrogen bonding polar interactions with R58 HC and L95 LC respectively, and I60, E61, Y82 and R109 form hydrophobic contacts (Table 1). R109 and K58 are involved in strong van der Waals interactions in the complex (Table 2) and form ion pairs with the K1-70 HC D54, D56 and D96 (Table 3).

In contrast, some TSHR amino acids that had no effect on the antagonist activity of K1-70 are involved in interactions within the complex. For example, TSHR R80 forms two salt bridges with K1-70 D50 LC, two water-mediated hydrogen bonds with Y99 HC, a nonhydrogen bonding polar interaction with N100 HC, a strong van der Waals interaction and two ion pair interactions with D50 LC. This shows that the absence of a detectable effect of a mutation in the TSHR on the actions of K1-70 on TSH stimulation does not necessarily indicate that there are no interactions involving this residue. In particular, a mutation may not show any effect if other interactions with the TSHR are not disrupted or are strong enough to compensate for the mutation (Lo Conte et al. 1999).
Wild-type and mutated K1-70 rFab preparations (N32A HC, R94D LC, W97A HC, Y99A HC and N100A HC) were expressed in Escherichia coli, and their activity was compared to the native K1-70 Fab produced by digestion of hybridoma K1-70 IgG. The activity of the K1-70 rFab showed a good agreement with the activity of the native Fab for inhibition of TSHbiotin binding and for inhibition of TSH-induced stimulation of cAMP production in CHO cells expressing the TSHR (Fig. 5A).

Western blotting showed that WT and mutated K1-70 $\mathrm{rFab}$ preparations contained $<5 \%$ smaller cleavage products, and the results of the Easy-Titre $(\mathrm{H}+\mathrm{L}) \operatorname{IgG}$ assay showed that WT and mutated K1-70 rFabs were expressed at a similar level $(3 \cdot 7-6 \cdot 0 \mu \mathrm{g} / \mathrm{ml})$. We compared the activity of K1-70 rFab in E. coli culture supernatant (concentration of $\mathrm{rFab}$ measured in the Pierce Easy-Titre Human IgG assay kit) to the activity of purified K1-70 rFab (concentration measured by $\mathrm{OD}_{280}$ ) using inhibition of TSH-biotin binding to the TSHR, and they were in good agreement (data not shown).

Mutation of K1-70 HC W97A and HC N100A greatly reduced the ability of K1-70 to inhibit TSH binding to TSHR-coated ELISA plate wells, i.e. 11.0 and $13 \cdot 8 \%$ inhibition respectively at $0 \cdot 1 \mu \mathrm{g} / \mathrm{ml}$ (compared with $93.6 \%$ by WT), and $17 \cdot 4$ and $37.8 \%$ respectively at $1 \mu \mathrm{g} / \mathrm{ml}$ (compared with $97 \cdot 2 \%$ for WT; Fig. 5B). K1-70 mutations LC R94D, HC Y99A and HC N32A also had an effect giving $62 \cdot 0,55 \cdot 2$ and $71 \cdot 7 \%$ inhibition of TSH binding at $0 \cdot 1 \mu \mathrm{g} / \mathrm{ml}$ respectively compared with $93 \cdot 6 \%$ in the case of WT (Fig. 5B).

The five K1-70 Fab mutations HC N100A, HC W97A, LC R94D, HC Y99A and HC N32A resulted in complete 

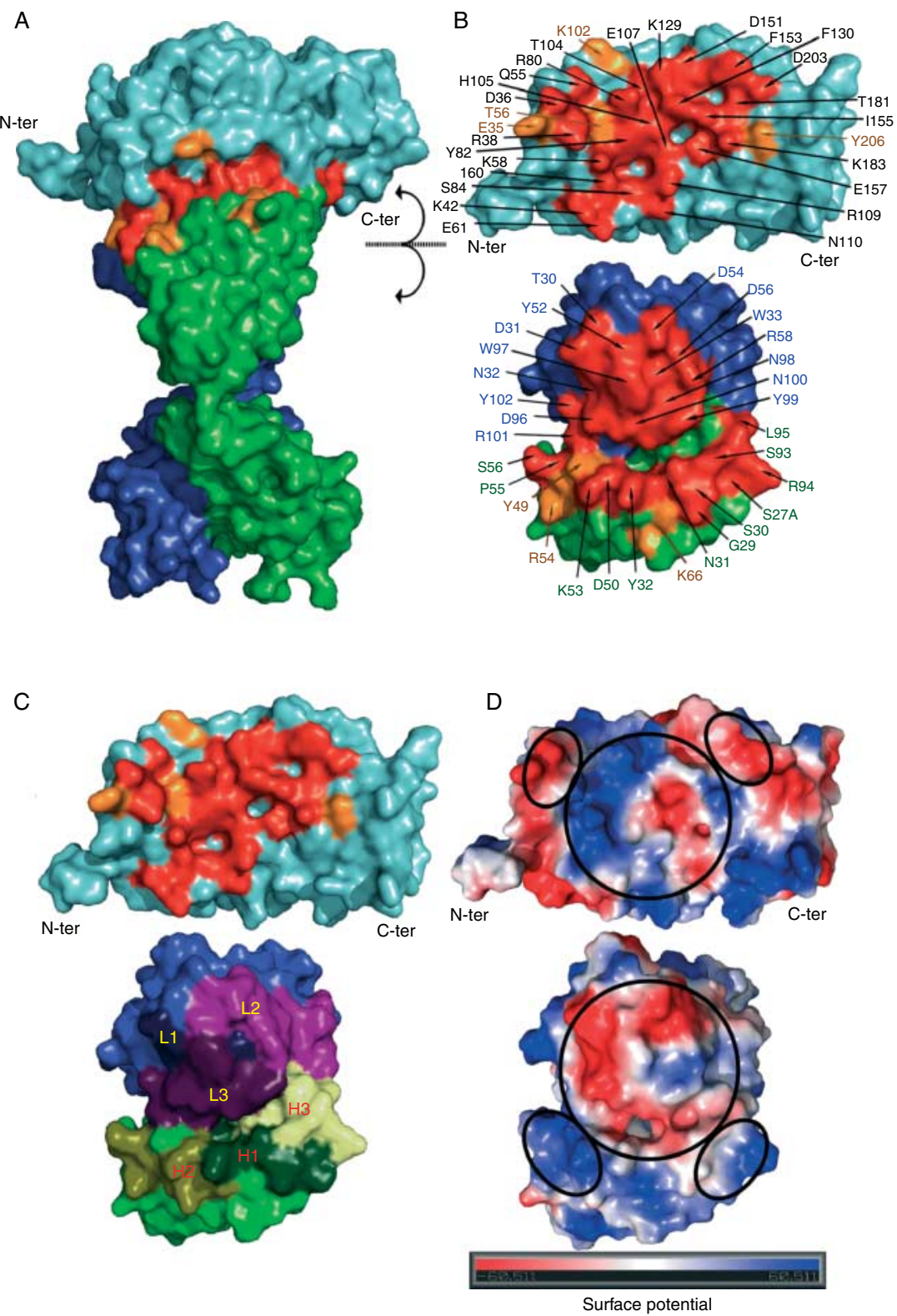

Figure 4 Interactions between K1-70 Fab and TSHR260. TSHR is shown in cyan, K1-70 heavy chain is in blue, and $\mathrm{K} 1-70$ light chain is in green. (A) The molecular surface of the TSHR260-K1-70 complex. TSHR residues are in cyan, K1-70 light chain is in green, and $\mathrm{K} 1-70$ heavy chain is in blue. (B) Opened up view of the interface area with residues of TSHR260 and K1-70 that are within 4.0 $\AA$ of each other highlighted in red and labelled, and those that interact only through water molecules are highlighted in orange and labelled. TSHR260 is shown in the top part of the figure and interacting residues are labelled. $\mathrm{K} 1-70$ is shown in the bottom part of the figure, the heavy chain residues are labelled in blue, and the light chain residues are labelled in green. (C) Hypervariable regions of K1-70 (bottom) are highlighted in different colours and labelled; TSHR (top) as in (B). (D) Electrostatic potential surface of TSHR260 (top) and K1-70 (bottom).

Complementary potential surfaces between TSHR and K1-70 are enclosed in circles.

The side chain of TSHR residue TSHR R250 is absent in the crystal structure and was modelled prior to generating the electrostatic surface potential of the TSHR LRD. 
abolition of the ability to inhibit TSH-induced stimulation of cAMP production giving $0-1.9 \%$ inhibition at $1 \mu \mathrm{g} / \mathrm{ml}$ compared with $75 \cdot 6 \%$ with WT K1-70 Fab (Fig. 5C). All five residues were observed to be involved in interactions with the TSHR in the crystal structure. Mutation of K1-70 HC W97 had the greatest effect with the ability of the mutant $(1 \mu \mathrm{g} / \mathrm{ml})$ to inhibit $\mathrm{TSH}$ binding to the receptor being $<20 \%$ inhibition (compared with over $90 \%$ inhibition of TSH binding with wild-type K1-70) and with no effect on TSHinduced cAMP production being demonstrable (compared with over $70 \%$ blocking of TSH-induced cAMP stimulation with wild-type K1-70; Fig. 5B and C). In the crystal structure, K1-70 HC W97 is involved in a hydrogen bond with TSHR E107, hydrophobic contacts with I60, Y82, S84, E107 and R109, and has strong van der Waals interactions (Table 2). HC N100 forms a hydrogen bond with TSHR Y82, a water-mediated bond with T56 and a non-hydrogen bonding polar interaction with R80.

\section{Comparison of TSHR-K1-70 Fab, TSHR-M22 Fab and TSHR-TSH complexes}

Analysis of the TSHR260-K1-70 Fab, TSHR260-M22 Fab (Sanders et al. 2007a) crystal structures and the TSH-TSHR comparative model (Núñez Miguel et al. 2008) allowed a comparison of the binding arrangements within the complexes (Table 5).

As described previously, binding of the human thyroid-stimulating autoantibody M22 mimics the

Table 2 Van der Waals interactions in the TSHR260-K1-70 complex. Van der Waals interactions were calculated as the accessible surface area (ASA) difference, for every amino acid, between the uncomplexed and complexed proteins. The ASA was calculated using the Lee and Richards algorithm developed by Richmond (Lee \& Richards 1971, Richmond 1984)

\begin{tabular}{lr}
\hline (A) TSHR260 residues involved in strong \\
(interaction surface area $>60 \AA^{2}$ ) van der \\
Waals interactions with K1-70 Fab \\
Arg80 \\
Phe153 & \\
Arg38 & $101 \cdot 5 \AA^{2}$ \\
Asp36 & $98 \cdot 3 \AA^{2}$ \\
Arg109 & $89 \cdot 9 \AA^{2}$ \\
Phe130 & $85 \cdot 8 \AA^{2}$ \\
Lys58 & $83 \cdot 5 \AA^{2}$ \\
Lys129 & $67 \cdot 2 \AA^{2}$ \\
(B) K1-70 Fab residues involved in strong & $62 \cdot 4 \AA^{2}$ \\
(interaction surface area > $60 \AA^{2}$ ) van der & $60 \cdot 8 \AA^{2}$ \\
Waals interactions with TSHR260 & \\
Trp97 HC & \\
Tyr99 HC & $117 \cdot 3 \AA^{2}$ \\
Arg94 LC & $109 \cdot 9 \AA^{2}$ \\
Tyr32 LC & $97 \cdot 9 \AA^{2}$ \\
Asp31 HC & $81 \cdot 9 \AA^{2}$ \\
Ser93 LC & $74 \cdot 4 \AA^{2}$ \\
Ser30 LC & $74 \cdot 2 \AA^{2}$ \\
\hline
\end{tabular}

binding of TSH to the TSHR (Núñez Miguel et al. 2009). The M22 LC mimics the binding of the TSH $\beta$ chain as both interact with the 1st, 2nd, 4th, 6th, 7th, 8th, 9th and 10th repeats of TSHR260. The M22 HC mimics the binding of the $\mathrm{TSH} \alpha$ chain and interacts with residues from the 1st through to the 7 th repeat (Table 5). In contrast, the K1-70 LC interacts with the 1 st through to the 8 th repeat, and the $\mathrm{HC}$ with the 1 st through to the 6 th repeat. Consequently, K1-70 does not mimic either the interactions of TSH or M22 binding to the TSHR (Table 5).

A comparison of interactions in the interfaces of the three complexes demonstrates the increased number of stronger interactions with the TSHR (salt bridges and hydrogen bonds) in the case of K1-70 and M22 compared with the TSHR-TSH complex (25, 22 and 7 hydrogen bonds and salt bridges respectively; Table 5 and Fig. 6). The amino acid residues on the concave surface of the TSHR leucine-rich domain (LRD) that interact with TSH in a comparative model (Núñez Miguel et al. 2008), the thyroid-stimulating autoantibody M22 in the crystal structure (Sanders et al. 2007a) and the TSHR blocking autoantibody K1-70 in the crystal structure are detailed in Fig. 6. There are 24, 25 and 21 residues on the TSHR260 that interact with K1-70, M22 and TSH respectively (Fig. 6). Although the binding sites of the three ligands on the TSHR concave surface overlap extensively, the actual contact of amino acids differ. There are 16 amino acids that interact with both K1-70 and M22, 12 amino acids that interact with both K1-70 and TSH, and 13 amino acids that interact with both M22 and TSH. Furthermore, ten TSHR amino acids R38, K58, R80, H105, R109, N110, F130, F153, I155 and E157 interact with all three ligands (Fig. 6).

\section{Discussion}

The availability of the crystal structure of K1-70 Fab (unbound) allowed us to compare the antigen-binding surfaces of this autoantibody with TSH-blocking (antagonist) activity and an autoantibody with thyroidstimulating (agonist) activity (M22; Sanders $e$ al. 2003, 2004). Both the K1-70 and M22 antigen-binding regions are rich in aromatic residues, and statistical analysis (Padlan 1990) has shown that tyrosines are three times more likely to be found in CDRs than in the frameworks of variable domains. The electrostatic surface potentials of both K1-70 and M22 TSHR-binding regions (Fig. 1) show highly irregular surfaces dominated by basic patches on one side and acidic patches on the other, although the distribution of the acidic and basic patches is virtually opposite for the two Fabs. In the case of M22, the negatively charged surface patches are formed predominantly by the LC residues, while on the 
Table 3 Ion pair interactions in the TSH receptor (TSHR) LRD-K1-70 Fab complex. The interaction strengths shown for comparison, are in Newtons and are calculated using an in house program (ELECINT, R Nunez Miguel, unpublished) taking $\varepsilon=1$ for electrostatic field calculation, and $\mathrm{pH}=7.4$ for the calculation of charges of side chain atoms of charged residues using the Henderson-Hasselbalch equation. Distances are between charged atoms

TSHR LRD

K1-70 Fab
Distance (strength)

\begin{tabular}{|c|}
\hline $\begin{array}{l}\mathrm{N} \\
\mathrm{N}) \\
\mathrm{N}) \\
\mathrm{N}\end{array}$ \\
\hline 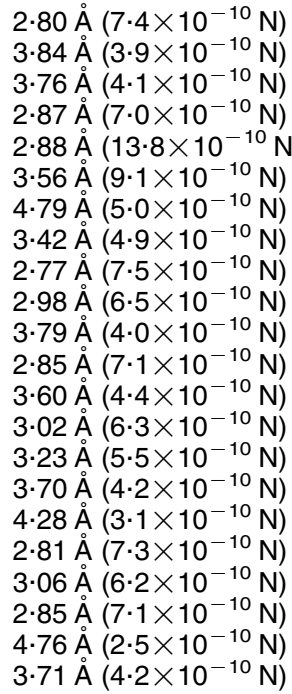 \\
\hline
\end{tabular}

surface of K1-70, the negatively charged residues are from the HC. The positively charged residues on the surface of M22 are from the HC, whereas in the case of K1-70, they are from the LC (Fig. 1). These observations are consistent with the important role for chargecharge interactions for binding of TSHR autoantibodies to the TSHR observed experimentally (Rees Smith et al. 1988).

The crystal structure of the TSHR in complex with $\mathrm{K} 1-70$ that we describe provides details, at the molecular level, of how a human autoantibody that blocks TSH (and thyroid-stimulating autoantibody) stimulation interacts with the TSHR. Furthermore, the interactions of the TSHR with the blocking autoantibody (K1-70) can be compared with the interactions of the TSHR with the thyroid-stimulating autoantibody (M22; Sanders et al. $2007 a, b)$.

A comparison of the TSHR260 structure when in complex with K1-70 or M22 shows an rmsd on all $\mathrm{C}_{\alpha}$ atoms between the structures of only $0 \cdot 51 \AA$ confirming the original solved structure of the TSHR LRD (Sanders et al. 2007a). Furthermore, the conformation of the TSHR260 structure shows no significant change between the complex with the blocking monoclonal antibody K1-70 and the complex with the stimulating monoclonal antibody M22. This observation suggests that the first step in the process of TSHR activation by stimulating antibodies (such as M22), i.e. ligand binding, does not involve a direct conformational change of the TSHR LRD, and how the binding of M22 (or other TSHR-activating ligands) causes receptor activation is not clear at present. It may be that initial binding to the TSHR LRD induces changes in the hinge region between the $\mathrm{LRD}$ and transmembrane domain (TMD). Then this conformational change could cause activation of the TMD (Kleinau et al. 2008, Mizutori et al. 2008, Mueller et al. 2008). Crystal structures of the complete TSHR extracellular domain in complex with M22 and in complex with K1-70 should provide key insights into the mechanism of activation.

In the asymmetric unit of the crystals, there was only one complex consisting of one molecule of TSHR260 
Table 4 Cyclic AMP production by TSH and blocking of TSH stimulation of cyclic AMP production by a human monoclonal autoantibody $\mathrm{K} 1-70$; effects in $\mathrm{CHO}$ cells transfected with mutated TSH receptor (TSHR) ${ }^{\mathrm{a}}$

\begin{tabular}{|c|c|c|}
\hline $\begin{array}{l}\text { TSHR } \\
\text { mutation }\end{array}$ & $\begin{array}{l}\text { Stimulation of } \\
\text { cyclic AMP } \\
\text { production } \\
\text { by TSH }\end{array}$ & $\begin{array}{l}\text { Blocking of } \\
\text { TSH-mediated } \\
\text { stimulation of cyclic } \\
\text { AMP production by } \\
\text { K1-70 lgG }\end{array}$ \\
\hline Wild type & +++++ & +++++ \\
\hline Asp43 Ala & +++ & +++++ \\
\hline Lys58 Ala & +++++ & + \\
\hline Ile60 Ala & +++++ & ++ \\
\hline Glu61 Ala & ++++ & +++ \\
\hline Arg80 Ala & +++++ & +++++ \\
\hline Tyr82 Ala & +++++ & +++ \\
\hline Thr104 Ala & +++++ & +++++ \\
\hline His105 Ala & +++++ & +++++ \\
\hline Glu107 Ala & +++ & +++++ \\
\hline Arg109 Ala & +++++ & + \\
\hline Lys129 Ala & +++++ & +++++ \\
\hline Phe130 Ala & +++++ & +++++ \\
\hline Phe134 Ala & +++++ & +++++ \\
\hline Asp151 Ala & +++++ & +++++ \\
\hline Glu157 Ala & ++ & +++++ \\
\hline Aso160 Ala & +++ & +++++ \\
\hline Glu178 Ala & ++++ & ++++ \\
\hline Lys183 Ala & +++++ & +++ \\
\hline Tyr185 Ala & ++++ & +++++ \\
\hline Asp203 Ala & ++++ & +++++ \\
\hline Tyr206 Ala & ++++ & +++++ \\
\hline Lys209 Ala & ++++ & +++++ \\
\hline Asp232 Ala & +++ & +++++ \\
\hline Gln235 Ala & +++++ & +++++ \\
\hline Lys250 Ala & +++++ & +++++ \\
\hline Glu251 Ala & +++++ & +++++ \\
\hline Arg255 Ala & +++++ & +++++ \\
\hline Thr257 Ala & +++++ & +++++ \\
\hline Trp258 Ala & +++++ & +++++ \\
\hline
\end{tabular}

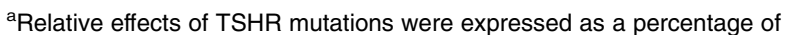
activity observed with wild type:,$+++++ 100 \%$ wild-type activity; ,$++++<100-80 \%$ wild-type activity;,$+++<80-60 \%$ wild-type activity;,$++<60-40 \%$ wild-type activity;,$+<40-20 \%$ wild-type activity.

bound to one molecule of K1-70 Fab. This was also the case in the TSHR260-M22 crystal structure (Sanders et al. 2007a), and consequently there was no evidence of dimer formation in either complex. In contrast, in the case of the FSHR-FSH complex crystal structure (solved at 2.9 А; Fan \& Hendrickson 2005), there were two complexes in the asymmetric unit. This difference could reflect differences in packing within the crystal, although FSHR-FSH complex dimerisation has been proposed (Fan \& Hendrickson 2005).

The higher $(1.9 \AA)$ resolution of the TSHR260-K1-70 structure provided details of the disulphide bond arrangements at the N-terminus of the TSHR for the first time (Fig. 2B). Disulphide bonds are present between the 1st and 2nd cysteines (C24 and C29) and between the 3rd and 4th cysteines (C31 and C41). Thus, $\mathrm{N}$-terminal cysteine disulphide bonding arrangements in the TSHR are different to that observed in the case of the FSHR crystal structure, where the 1st and 3rd cysteines (C18 and C25) and the 2nd and 4th cysteines (C23 and C32) are disulphide bonded (Fan \& Hendrickson 2005). The TSHR has a three amino acid insertion between TSHR C31 and C41 when compared with the FSHR sequence, and consequently bonding between C29 and C41 of the TSHR would be entropically less favourable. Based on TSHR mutagenesis studies reported previously (Chen et al. 2001), it was proposed that C41 was paired to either C29 or C31, but our crystal structure now provides molecular level detail of the cysteine pairing at the N-terminus of the TSHR including C41 paired to C31.

In the crystal structure, K1-70 shows no interaction with the extreme N-terminus of the TSHR LRD, which contains the bonded cysteines. Analysis of the interaction of M22 with the TSHR (using the TSHR LRD structure from the complex solved at $1.9 \AA$ ) also showed that there were no M22 interactions involving the extreme $\mathrm{N}$-terminal region of the TSHR LRD. These observations suggest that the TSHR region containing the four disulphide-bonded cysteines does not have a major role in TSHR autoantibody binding and most likely acts as a protective N-terminal cap aiding stability, preventing degradation and keeping the correct conformation of the receptor. This is not consistent with previous studies, which have concluded that the TSHR N-terminal region is part of a highly conformational epitope for thyroid-stimulating autoantibodies (Chen et al. 2001, Chazenbalk et al. 2004).

The crystal structure of the complex shows that the K1-70 Fab clasps the concave surface of the TSHR LRD in a similar way to M22 Fab but is in approximately the opposite orientation (i.e. rotated by $\sim 155^{\circ}$ on the TSHR helical tube axis; Fig. 3). However, K1-70 binds more N-terminal on the TSHR interacting with an extensive surface of the receptor between amino acid D36 and D203, while the binding site of M22 extends further towards the C-terminus and spans the region between TSHR amino acids D36 and N256 (Fig. 3). The concave surface of the TSHR that interacts with K1-70 is extensive ( $\triangle$ ASA $2565 \cdot 4 \AA^{2}$ ) and is greater than that typically observed for an antibody-antigen interface (Jones \& Thornton 1996, Lo Conte et al. 1999). This observation together with the presence of a large number of hydrogen bonds, salt bridges and van der Waals interactions in the interface reflects the high binding affinity of K1-70 for the TSHR. Similar types and levels of interaction are observed for M22 (Sanders et al. 2007a). In contrast, there are only seven hydrogen bonds and salt bridges in the TSHR260-TSH complex (comparative model Núñez Miguel et al. 2008) compared with 25 and 22 in the complex of TSHR260 with K1-70 or M22 respectively, and this reflects the 
differences in binding affinity of these three ligands to the TSHR.

Comparison of the structures of K1-70 Fab unbound and bound to the TSHR260 indicates that there is essentially no movement in the atoms of K1-70 residues upon binding to the TSHR, which is consistent with a 'lock and key' mode of binding (Supplementary Figure 1). This was also observed in the case of M22 binding to the TSHR (Sanders et al. 2007a), and

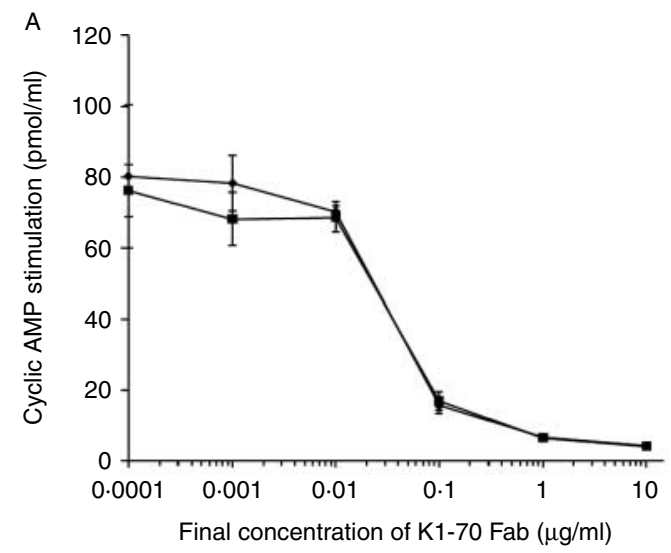

B
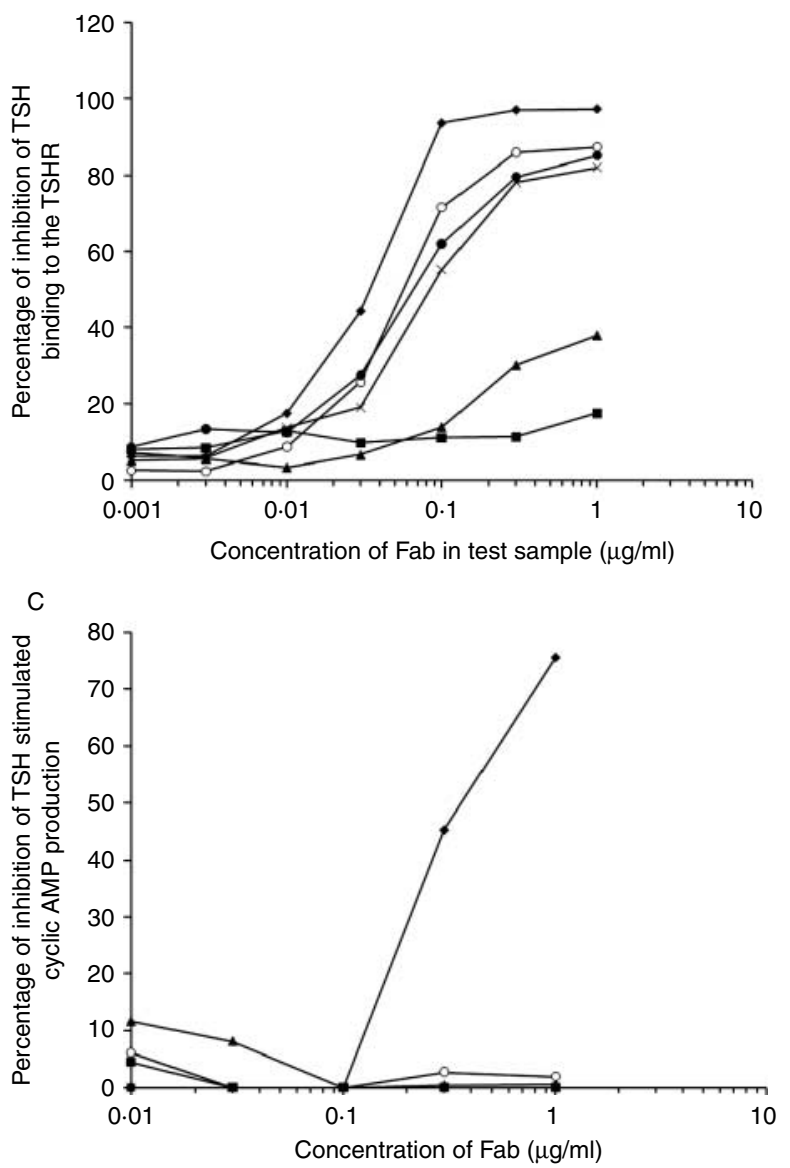

consequently, loss of free energy through induction of a conformational change in either K1-70 or M22 does not occur during binding, and this is also consistent with the observed high affinity of the autoantibodyreceptor interaction (McLachlan \& Rapoport 1996, Sanders et al. 2003, 2004, 2008, Nakatake et al. 2006, Morganthaler et al. 2007, Thorpe \& Brooks 2007, Rees Smith et al. 2009, Evans et al. 2010). In contrast to autoantibody binding to the TSHR, considerable conformational changes occur in FSH on binding to the FSH receptor (Fan \& Hendrickson 2005).

Results from TSH binding and cAMP stimulation assays using mutated TSHR or K1-70 Fab provided experimental evidence confirming interactions observed in the crystal structure. Mutations of different TSHR residues that showed strong interactions in the structure influenced K1-70 activity, indicating that the binding arrangements in the complex reflect the interactions of K1-70 with intact, functionally active TSHR at least in vitro, and it is most likely that similar interactions take place when the TSHR and K1-70 combine in vivo.

However, due to the large interfaces of the interacting surfaces of all three ligands (K1-70, M22 and TSH; 2565, 2514 and $2533 \AA^{2}$ respectively) with the TSHR, mutation of some interacting residues may not show an effect on binding and/or biological activity (Lo Conte et al. 1999). For example, TSHR R80 forms several interactions with K1-70 in the complex, but mutation of this residue does not affect K1-70 activity. This is also the case for the mutation of TSHR E251 to alanine (Sanders et al. 2006, Chen et al. 2010), which interacts with TSH K44 $\beta$ in the TSH-TSHR comparative model (Núñez Miguel et al. 2008). The salt bridge formed between TSHR E251 and TSH K44 $\beta$ would not be formed in the case of TSHR E251A mutation; however, as this interaction is on the periphery of the interface, the mutation would be unlikely to affect TSH

Figure 5 (A) Effects of $\mathrm{K} 1-70$ recombinant Fab (filled square)- and native hybridoma (filled diamond)-produced K1-70 Fab preparations on the inhibition of porcine TSH (pTSH) stimulation of cAMP in $\mathrm{CHO}$ cells expressing wild-type TSH receptor (TSHR). Final concentration of $\mathrm{pTSH}$ was $3 \mathrm{ng} / \mathrm{ml}$ and gave $76 \cdot 5$ $\pm 7 \cdot 2 \mathrm{pmol} / \mathrm{ml}$ cAMP production in the absence of $\mathrm{K} 1-70$ preparations. Mean of triplicate determinations. (B) Effects of wild-type and mutated K1-70 recombinant Fabs on the inhibition of TSH binding to TSHR coated onto ELISA plates. Test samples $(75 \mu \mathrm{l})$ were added to $75 \mu \mathrm{l}$ start buffer in each well. Wild type (filled diamond), W97A HC (filled square), N100A HC (filled triangle), Y99A HC (multiplication sign), N32A HC (open circle), R94D LC (filled circle). (C) Effects of wild-type and mutated K1-70 recombinant Fabs on the inhibition of TSH stimulation of cyclic AMP production. Final concentration of TSH was $3 \mathrm{ng} / \mathrm{ml}$. Wild type (filled diamond), W97A HC (filled square), N100A HC (filled triangle), Y99A HC (multiplication sign), N32A HC (open circle), R94D LC (filled circle). 
Table 5 Interactions between the TSH receptor (TSHR) and TSH, M22 and K1-70

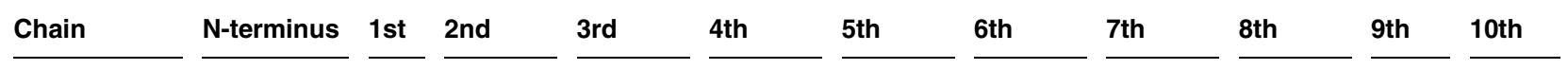

(A) Number of residues from TSH ( $\alpha$ and $\beta$ chains), M22 (heavy and light chains) and K1-70 (heavy and light chains) that interact with residues of the TSH receptor (TSHR) leucine-rich repeats

\begin{tabular}{|c|c|c|c|c|c|c|c|c|c|c|c|}
\hline $\mathrm{TSH} \alpha$ & - & 1 & 1 & 6 & 9 & 6 & 8 & 2 & - & - & - \\
\hline $\mathrm{TSH} \beta$ & - & 1 & 3 & - & 4 & - & 3 & 1 & 4 & 1 & 3 \\
\hline M22 LC & - & 1 & 1 & - & 3 & - & 3 & 2 & 7 & 2 & 6 \\
\hline M22 HC & - & 4 & 2 & 4 & 4 & 7 & 7 & 3 & - & - & - \\
\hline K1-70 LC & - & 3 & 1 & 2 & 1 & 3 & 5 & 3 & 1 & - & - \\
\hline K1-70 HC & - & 6 & 5 & 4 & 8 & 2 & 2 & - & - & - & - \\
\hline Complex & & Salt bridges & 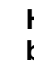 & & Ion pairs & & Polar & & nduced & Hy & nobic \\
\hline
\end{tabular}

(B) Number and type of interactions in the interface of the TSHR260-K1-70 complex (crystal structure solved at 1.9 A), TSHR260-M22 (crystal structure solved at 2.55 $\AA$ ) and the TSHR-TSH complex (comparative model)

$\begin{array}{lrrrrrr}\text { K1-70-TSHR } & 11 & 14 & 8 & 11 & 48 & 19 \\ \text { M22-TSHR } & 8 & 14 & 10 & 17 & 36 & 14 \\ \text { TSH-TSHR } & 4 & 3 & 7 & 18 & 36 & 22\end{array}$

binding and stimulating activities (Sanders et al. 2006, Chen et al. 2010).

Mutation studies (Sanders et al. 2006) identified TSHR R255 as important for the activity of the stimulating autoantibody M22 and patient serumstimulating autoantibodies. However, mutation of TSHR R255 did not affect the TSH antagonist (blocking) activity of K1-70 and has also been reported to be unimportant for the antagonist activity of patient serum autoantibodies (Sanders et al. 2006). Furthermore, TSHR R255A has no effect on the antagonist activities of the mouse MAb-B2 (Sanders et al. 2005, 2006) and the human autoantibody 5C9 (Sanders et al. 2008; both with TSH and TSHR autoantibody antagonist activity). In addition, a bridge-type ELISA using TSHR260-AP and TSHR containing R255D mutation can distinguish between the blocking-type monoclonal autoantibody K1-70, which binds to the mutated TSHR, and the stimulating-type monoclonal autoantibody M22, which shows drastically reduced binding compared with the WT TSHR. In the crystal structure, K1-70 Fab does not interact with TSHR R255, and this is in agreement with the experimental studies described in this study and reported previously (Sanders et al. 2006, Rees Smith et al. 2009). In contrast, TSHR C-terminal patches are involved in electrostatic interactions with thyroid-stimulating autoantibody M22, and R255 is the most C-terminal TSHR260 residue showing charge-charge interactions with M22 LC D60 (Sanders et al. 2007a).

The exact location of the binding sites for blocking autoantibodies and stimulating autoantibodies on the TSHR molecule has been controversial. Experimental evidence from our laboratory (Sanders et al. 1999, 2002,
2006) and other laboratories (Nagayama et al. 1991, Rapoport et al. 1998, Morganthaler et al. 2003, 2007) suggests that the epitopes for both types of autoantibodies overlap extensively. In contrast, other reports based on studies with TSHR chimeras concluded that the major binding sites for thyroid-stimulating autoantibodies were located at the N-terminus of the TSHR amino acids 9-165 (Tahara et al. 1991, 1997, Kosugi et al. 1993, Kim et al. 1996, 1997, Minich \& Loos 2000, Kung et al. 2001, Minich et al. 2004), while the epitopes of blocking-type autoantibodies were more towards the TSHR C-terminus (aa 261-370; Tahara et al. 1997, Minich \& Loos 2000, Kung et al. 2001). However, the binding arrangements observed in the crystal structure of the TSHR complexed with a blocking-type autoantibody (K1-70) and a stimulating-type autoantibody (M22) clearly show that the binding sites of the two antibodies overlap considerably with K1-70 forming strong interactions with TSHR260 (aa 22-260), even though the previously suggested important blocking antibody epitope (aa 261-370) (Tahara et al. 1997, Minich \& Loos 2000, Kung et al. 2001, Loos et al. 2007) is missing entirely. Furthermore, both K1-70 and M22 bind strongly in ELISAs based on TSHR260 (Rees Smith et al. 2009). However, there has been one report (Schwarz-Lauer et al. 2002) in which the TSHR-blocking activity of one patient serum could not be absorbed by purified TSHR289 (aa 22-289), even though the blocking antibodies in a different serum and all the stimulating antibodies studied were absorbed. This could indicate that occasional patient autoantibodies with blocking activity might bind outside the TSHR260 region. However, in a detailed study of the interaction of over 50 patient serum TSHR autoantibodies with 


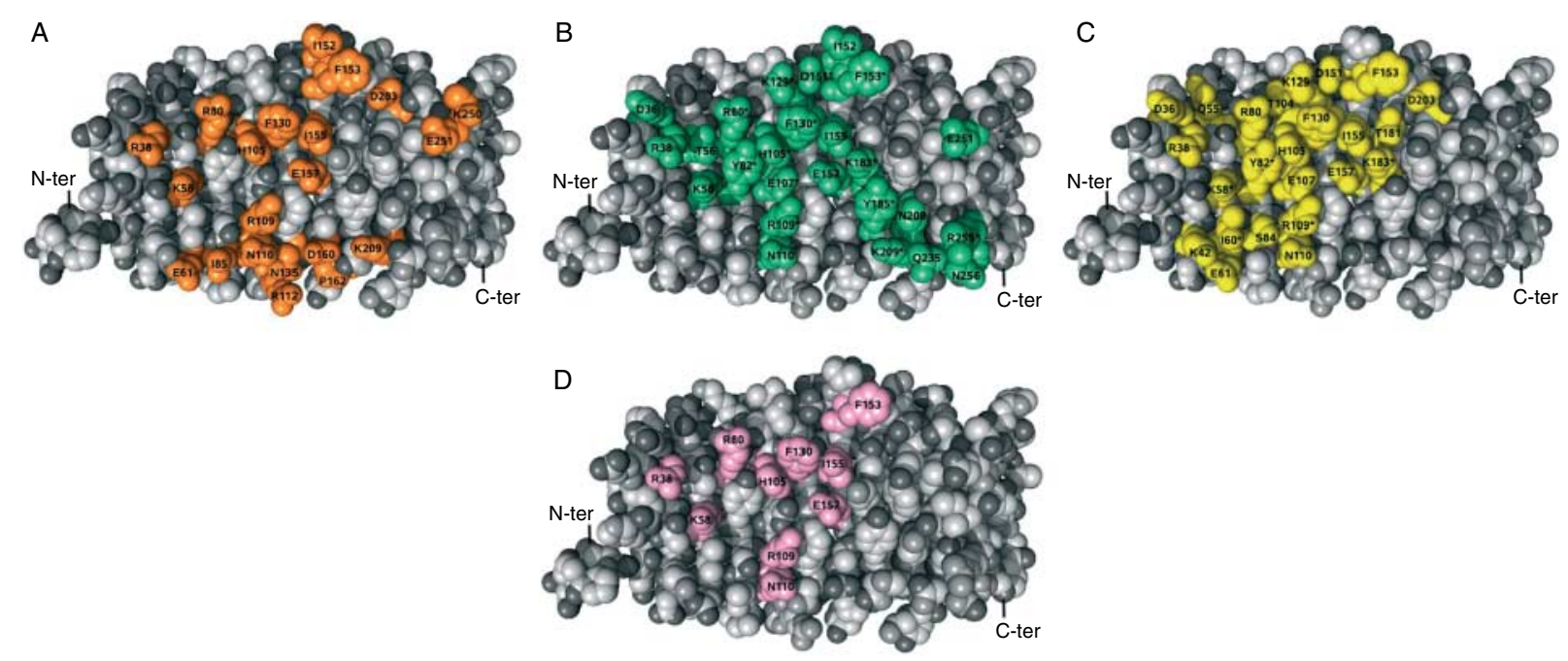

Figure 6 Space-fill representation of the TSH receptor leucine-rich repeat domain (TSHR LRD) interactive surface (based on the crystal structure of the TSHR260 in complex with K1-70 solved at 1.9 A resolution). The $\mathrm{N}$-terminus and the C-terminus are indicated. (A) TSHR residues that interact with TSH in our TSH-TSHR260 comparative model are shown in orange. (B) TSHR residues that interact with thyroid-stimulating autoantibody M22 in the TSHR260-M22 crystal structure are shown in green. (C) TSHR residues that interact with TSHR-blocking autoantibody K1-70 in the TSHR260-K1-70 crystal structure are shown in yellow. (D) TSHR residues that interact with all three ligands TSH, M22 and K1-70 are shown in pink.

TSHR260, all sera were found to interact strongly with this receptor preparation (Rees Smith et al. 2009). In addition, five sera in which blocking activity was established and five sera in which stimulating activity was established bound strongly to TSHR260.

A comparison of the interactions between the TSHR and the TSHR autoantibodies K1-70 and M22 and the hormone TSH was carried out using the respective crystal structures and a comparative model of the TSHR-TSH complex (Sanders et al. 2007a, Núñez Miguel et al. 2008). TSHR autoantibodies with different biological activities interact with the same region of the TSHR (the concave surface of the TSHR LRD) as TSH itself. Thus, the crystal structures provide further evidence for the overlap of the binding sites for TSHR autoantibodies and TSH observed in experimental studies (Rees Smith et al. 1988, Rapoport et al. 1998, Sanders et al. 1999, 2002, 2006, Morganthaler et al. 2003, 2007, Costagliola et al. 2004, Latif et al. 2009). Furthermore, the binding arrangements in the three complexes show important differences in the types of interactions present. In particular, interactions of K1-70 and M22 with the TSHR involve a greater number of strong interactions (hydrogen bonds and salt bridges) than in the case of TSH-TSHR binding. Also the interactions between FSH and FSHR in the crystal structure of the complex involve only six hydrogen bonds and salt bridges (Fan \& Hendrickson 2005, Núñez Miguel et al. 2008). Thus, TSH or FSH binding to their respective receptors favours hydrophobic interactions, while binding of autoantibodies to the
TSHR depends on stronger electrostatic interactions. The differences observed in the interactions in the complexes of the TSHR with TSH and with autoantibodies may reflect differences in how these complexes evolved. From an evolutionary perspective, it would be expected that hormone-receptor interactions have undergone evolutionary optimisations over a very long time that would include a trade-off between specificity of binding and effective biological function. In contrast, antibody-antigen interactions are subject to selection principally driven by binding affinity in addition to binding specificity (Jones \& Thornton 1996, Lo Conte et al. 1999, Thorpe \& Brooks 2007).

The crystal structures also showed that although M22 and K1-70 both bind to the concave surface of TSHR260 with considerable overlap, there are important differences. K1-70 interacts less with the C-terminus than TSH or M22. Furthermore, K1-70 HC and LC interactions with the TSHR LRD do not mimic the interactions of $\mathrm{TSH} \alpha$ and $\operatorname{TSH} \beta$ chains, while the interactions of M22 HC and LC do (Núñez Miguel et al. 2008). These arrangements most likely reflect the differences in the biological activity of the three ligands, and the observation of molecular mimicry between TSH and M22 (Núñez Miguel et al. 2008) but not between TSH and K1-70 provides a new perspective on the evolution of autoimmune responses in Graves' disease (Bork et al. 1993, Sanders et al. 2007a, Núñez Miguel et al. 2009).

Irrespective of the differences in the type of interactions and the regions on the TSHR LRD concave surface involved in binding to M22, K1-70 and TSH, all 
three ligands form important interactions with the same ten TSHR residues (Fig. 6). This observation may be helpful in further understanding the molecular basis of binding between the TSHR and ligands with different biological activities and may lead to the development of new therapies for autoimmune diseases involving the TSHR.

Overall, the availability of high resolution crystal structures of the TSHR in complex with blocking-type and stimulating-type human monoclonal autoantibodies provides a foundation for developing new strategies to understand and control TSHR activation and the autoimmune response to the TSHR.

\section{Supplementary data}

This is linked to the online version of the paper at http://dx.doi.org/ 10.1530/JME-10-0127.

\section{Declaration of interest}

RSR Ltd is a developer of in vitro medical diagnostics including kits for measuring thyroid autoantibodies. All authors are employees of RSR Ltd.

\section{Funding}

The work was funded by RSR Ltd.

\section{Acknowledgements}

Some of the crystallisation trials, X-ray diffraction data collection and structure solving were carried out at Biofocus (Saffron Walden, UK) by Dr Philip Leonard.

\section{References}

Birk HW \& Koepsell H 1987 Reaction of monoclonal antibodies with plasma membrane proteins after binding on nitrocellulose: renaturation of antigenic sites and reduction of non-specific antibody binding. Analytical Biochemistry 164 12-22. (doi:10.1016/ 0003-2697(87)90360-5)

Bolton J, Sanders J, Oda Y, Chapman C, Konno R, Furmaniak J \& Rees Smith B 1999 Measurement of thyroid-stimulating hormone receptor autoantibodies by ELISA. Clinical Chemistry 45 2285-2287.

Bork P, Sander C \& Valencia A 1993 Convergent evolution of similar enzymatic function on different protein folds: the hexokinase, ribokinase and galactokinase families of sugar kinases. Protein Science 2 31-40. (doi:10.1002/pro.5560020104)

Chazenbalk GD, Latrofa F, McLachlan SM \& Rapoport B 2004 Thyroid stimulation does not require antibodies with identical epitopes but does involve recognition of a critical conformation at the $\mathrm{N}$ terminus of the thyrotropin receptor A-subunit. Journal of Clinical Endocrinology and Metabolism 89 1788-1793. (doi:10.1210/jc.2003-031554)

Chen CR, Tanaka K, Chazenbalk GD, McLachlan SM \& Rapoport B 2001 A full biological response to autoantibodies in Graves' disease requires a disulfide-bond loop in the thyrotropin $\mathrm{N}$-terminus homologous to a laminin EGF-like domain. Journal of Biological Chemistry 276 14767-14772. (doi:10.1074/jbc.M008001200)
Chen CR, McLachlan SM \& Rapoport B 2010 Thyrotropin (TSH) receptor residue E251 in the extracellular leucine-rich repeat domain is critical for linking TSH binding to receptor activation. Endocrinology 151 1940-1947. (doi:10.1210/en.2009-1430)

Collaborative Computational project, number 41994 The CCP4 Suite: Programmes for protein Crystallography. Acta Crystallographica D50 760-763.

Costagliola S, Bonomi M, Morganthaler NG, Van Durme J, Panneels V, Refetoff S \& Vassart G 2004 Delineation of the discontinuousconformational epitope of a monoclonal antibody displaying full in vitro and in vivo thyrotropin activity. Molecular Endocrinology 18 3020-3034. (doi:10.1210/me.2004-0231)

DeLano WL 2002 The Pymol molecular graphics system. DeLano Scientific, San Carlos, CA. http://pymol.sourceforge.net/.

Emsley P, Lohkamp B, Scott W \& Cowtan K 2010 Features and developments of COOT. Acta Crystallographica D66 486-501. (doi:10.1107/S0907444910007493)

Evans M, Sanders J, Tagami T, Sanders P, Young S, Roberts E, Wilmot J, $\mathrm{Hu}$ X, Kabelis K, Clark J et al. 2010 Monoclonal autoantibodies to the TSH receptor, one with stimulating activity and one with blocking activity, obtained from the same blood sample. Clinical Endocrinology 73 404-412. (doi:10.1111/j.1365-2265.2010.03831.x)

Fan QR \& Hendrickson WA 2005 Structure of human folliclestimulating hormone in complex with its receptor. Nature $\mathbf{4 3 3}$ 269-277. (doi:10.1038/nature03206)

Jeffreys J, Depraetere H, Sanders J, Oda Y, Evans M, Kiddie A, Richards T, Furmaniak J \& Rees Smith B 2002 Characterization of the thyrotropin binding pocket. Thyroid 12 1051-1061. (doi:10. 1089/105072502321085144)

Jones S \& Thornton JM 1996 Principles of protein-protein interactions. PNAS 93 13-20. (doi:10.1073/pnas.93.1.13)

Kim WB, Cho BY, Park HY, Lee HK, Kohn LD, Tahara K \& Koh C 1996 Epitopes for thyroid-stimulating antibodies in Graves' sera: a possible link of heterogeneity to differences in response to antithyroid drug treatment. Journal of Clinical Endocrinology and Metabolism 81 1758-1767. (doi:10.1210/jc.81.5.1758)

Kim WB, Chung HK, Lee HK, Kohn LD, Tahara K \& Cho BY 1997 Changes in epitopes for thyroid-stimulating antibodies in Graves' disease sera during treatment of hyperthyroidism: therapeutic implications. Journal of Clinical Endocrinology and Metabolism 82 1953-1959. (doi:10.1210/jc.82.6.1953)

Kleinau G, Jaeschke H, Mueller S, Raaka BM, Neumann S, Paschke R \& Krause G 2008 Evidence for cooperative signal triggering at the extracellular loops of the TSH receptor. FASEB Journal 22 2798-2808. (doi:10.1096/fj.07-104711)

Kosugi S, Ban T \& Kohn LD 1993 Identification of thyroid antibodyspecific interaction sites in the N-terminal region of the thyrotropin receptor. MolecularEndocrinology 7 114-130. (doi:10.1210/me.7.1.114)

Kung AWC, Lau KS \& Kohn LD 2001 Epitope mapping of TSH receptor-blocking antibodies in Graves' disease that appear during pregnancy. Journal of Clinical Endocrinology and Metabolism 86 3647-3653. (doi:10.1210/jc.86.8.3647)

Laemmli UK 1970 Cleavage of structural proteins during the assembly of the head of bacteriophage T5. Nature 227 680-684. (doi:10.1038/ 227680a0)

Laskowski RA, MacArthur MW, Moss DS \& Thornton JM 1993 PROCHECK - a program to check the stereochemical quality of protein structures. Journal of Applied Crystallography 26 283-291. (doi:10.1107/S0021889892009944)

Latif R, Morshed SA, Zaidi M \& Davies TF 2009 The thyroid stimulating hormone receptor: impact of thyroid-stimulating hormone and thyroid stimulating hormone receptor antibodies on multimerisation, cleavage, and signaling. Endocrinology and Metabolism Clinics of North America 38 319-341. (doi:10.1016/j.ecl.2009.01.006)

Lee B \& Richards FM 1971 The interpretation of protein structures: estimation of static accessibility. Journal of Molecular Biology $\mathbf{5 5}$ 379-400. (doi:10.1016/0022-2836(71)90324-X) 
Lo Conte L, Chothia C \& Janin J 1999 The atomic structure of proteinprotein recognition sites. Journal of Molecular Biology 285 2177-2198. (doi:10.1006/jmbi.1998.2439)

Loos U, Franz C, Minich WB \& Büsselmann I 2007 Direct assay of TSH receptor autoantibodies causing Graves' disease correlates with the clinical diagnosis closer than assays based on TSH displacement. Hormone Research 68 (Supplement 3) 21-91.

McKenzie JM \& Zakarija M 1992 Fetal and neonatal hyperthyroidism and hypothyroidism due to maternal TSH receptor antibodies. Thyroid 2 155-159. (doi:10.1089/thy.1992.2.155)

McLachlan SM \& Rapoport B 1996 Monoclonal, human autoantibodies to the TSH receptor - the holy grail and why are we looking for it? Journal of Clinical Endocrinology and Metabolism 81 3152-3154. (doi:10.1210/jc.81.9.3152)

Minich WB \& Loos U 2000 Detection of functionally different types of pathological autoantibodies against thyrotropin receptor in Graves' patients sera by luminescent immunoprecipitation analysis. Experimental and Clinical Endocrinology and Diabetes 108 110-119. (doi:10.1055/s-2000-5804)

Minich WB, Lenzner C, Bergmann A \& Morgenthaler NG 2004 A coated tube assay for the detection of blocking thyrotropin receptor autoantibodies. Journal of Clinical Endocrinology and Metabolism 89 352-356. (doi:10.1210/jc.2003-030823)

Mizutori Y, Chen CR, McLachan SM \& Rapoport B 2008 The thyrotropin receptor hinge region is not simply a scaffold for the leucine-rich domain but contributes to ligand binding and signal transduction. Molecular Endocrinology 22 1171-1182. (doi:10.1210/ me.2007-0407)

Morganthaler NG, Minich WB, Willnich M, Bogusch T, Hollidt JM, Weglohner W, Lenzner C \& Bergmann A 2003 Affinity purification and diagnostic use of TSH receptor autoantibodies from human serum. Molecular and Cellular Endocrinology 212 73-79. (doi:10.1016/ j.mce.2003.09.018)

Morganthaler NG, Ho SC \& Minich WB 2007 Stimulating and blocking thyroid stimulating hormone (TSH) receptor autoantibodies from patients with Graves' disease and autoimmune hypothyroidism have very similar concentration, TSH receptor affinity, and binding sites. Journal of Clinical Endocrinology and Metabolism 92 1058-1065. (doi:10.1210/jc.2006-2213)

Mueller S, Kleinau G, Jaeschke H, Paschke R \& Krause G 2008 Extended hormone binding site of the human thyroid stimulating hormone receptor. Journal of Biological Chemistry 283 18048-18055. (doi:10.1074/jbc.M800449200)

Nagayama Y, Wadsworth HL, Russo D, Chazenbalk GD \& Rapoport B 1991 Binding domains of stimulatory and inhibitory thyrotropin (TSH) receptor autoantibodies determined with chimeric TSHlutropin/chorionic gonadotropin receptors. Journal of Clinical Investigation 88 336-340. (doi:10.1172/JCI115297)

Nakatake N, Sanders J, Richards T, Burne P, Barrett C, Dal Pra C, Presotto F, Betterle C, Furmaniak J \& Rees Smith B 2006 Estimation of serum TSH receptor autoantibody concentration and affinity. Thyroid 16 1077-1084. (doi:10.1089/thy.2006.16. 1077)

Núñez Miguel R, Sanders J, Chirgadze DY, Blundell TL, Furmaniak J \& Rees Smith B 2008 FSH and TSH binding to their respective receptors: similarities, differences and implication for glycoprotein hormone specificity. Journal of Molecular Endocrinology 41 145-164. (doi:10.1677/JME-08-0040)

Núñez Miguel R, Sanders J, Chirgadze DY, Furmaniak J \& Rees Smith B 2009 Thyroid stimulating autoantibody M22 mimics TSH binding to the TSH receptor leucine rich domain: a comparative structural study of protein-protein interactions. Journal of Molecular Endocrinology 42 381-395. (doi:10.1677/JME-08-0152)

Padlan EA 1990 On the nature of antibody combining sites. Unusual structural features that may confer on these sites an enhanced capacity for binding ligands. Proteins 7 112-124. (doi:10.1002/prot. 340070203)
Rapoport B, Chazenbalk GD, Jaume JC \& McLachlan SM 1998 The thyrotropin (TSH) receptor: interaction with TSH and autoantibodies. Endocrine Reviews 19 673-716. (doi:10.1210/er.19.6.673)

Rees Smith B, McLachlan SM \& Furmaniak J 1988 Autoantibodies to the thyrotropin receptor. Endocrine Reviews 9 106-121. (doi:10. 1210/edrv-9-1-106)

Rees Smith B, Sanders J \& Furmaniak J 2007 TSH receptor antibodies. Thyroid 17 923-938. (doi:10.1089/thy.2007.0239)

Rees Smith B, Sanders J, Evans M, Tagami T \& Furmaniak J 2009 TSH receptor-autoantibody interactions. Hormone and Metabolic Research 41 448-455. (doi:10.1055/s-0029-1220913)

Richmond TJ 1984 Solvent accessible surface area excluded volume in proteins. Analytical equations for overlapping spheres and implications for the hydrophobic effect. Journal of Molecular Biology 178 63-89. (doi:10.1016/0022-2836(84)90231-6)

Sanders J, Oda Y, Roberts SA, Maruyama M, Furmaniak J \& Rees Smith B 1997 Understanding the thyrotropin receptor function-structure relationship. Baillière's Clinical Endocrinology and Metabolism 11 451-479. (doi:10.1016/S0950-351X(97)80693-3)

Sanders J, Oda Y, Roberts S, Kiddie A, Richards T, Bolton J, McGrath V, Walters S, Jaskólski D, Furmaniak J et al. 1999 The interaction of TSH receptor autoantibodies with ${ }^{125}$ I-labelled TSH receptor. Journal of Clinical Endocrinology and Metabolism 84 3797-3802. (doi:10.1210/jc.84.10.3797)

Sanders J, Jeffreys J, Depraetere H, Richards T, Evans M, Kiddie A, Brereton K, Groenen M, Oda Y, Furmaniak J et al. 2002 Thyroid stimulating monoclonal antibodies. Thyroid 12 1043-1050. (doi:10. $1089 / 105072502321085135)$

Sanders J, Evans M, Premawardhana LDKE, Depraetere H, Jeffreys J, Richards T, Furmaniak J \& Rees Smith B 2003 Human monoclonal thyroid stimulating autoantibody. Lancet 362 126-128. (doi:10. 1016/S0140-6736(03)13866-4)

Sanders J, Jeffreys J, Depraetere H, Evans M, Richards T, Kiddie A, Brereton K, Premawardhana LD, Chirgadze DY, Núñez Miguel R et al. 2004 Characteristics of a human monoclonal autoantibody to the thyrotropin receptor: sequence structure and function. Thyroid 14 560-570. (doi:10.1089/1050725041692918)

Sanders J, Allen F, Jeffreys J, Bolton J, Richards T, Depraetere H, Nakatake N, Evans M, Kiddie A, Premawardhana LDKE et al. 2005 Characteristics of a monoclonal antibody to the thyrotropin receptor that acts as a powerful thyroid-stimulating autoantibody antagonist. Thyroid 15 672-682. (doi:10.1089/thy.2005.15.672)

Sanders J, Bolton J, Sanders P, Jeffreys J, Nakatake N, Richards T, Evans M, Kiddie A, Summerhayes S, Roberts E et al. 2006 Effects of TSH receptor mutations on binding and biological activity of monoclonal antibodies and TSH. Thyroid 16 1195-1206. (doi:10. $1089 /$ thy.2006.16.1195)

Sanders J, Chirgadze DY, Sanders P, Baker S, Sullivan A, Bhardwaja A, Bolton J, Reeve M, Nakatake N, Evans M et al. 2007a Crystal structure of the TSH receptor in complex with a thyroid-stimulating autoantibody. Thyroid 17 395-410. (doi:10.1089/thy.2007.0034)

Sanders J, Núñez Miguel R, Bolton J, Bhardwaja A, Sanders P, Nakatake N, Evans M, Furmaniak J \& Rees Smith B $2007 b$ Molecular interactions between the TSH receptor and a thyroid-stimulating monoclonal autoantibody. Thyroid 17 699-706. (doi:10.1089/thy.2007.0041)

Sanders J, Evans M, Betterle C, Sanders P, Bhardwaja A, Young S, Roberts E, Wilmot J, Richards T, Kiddie A et al. 2008 A human monoclonal autoantibody to the thyrotropin receptor with thyroidstimulating blocking activity. Thyroid 18 735-746. (doi:10.1089/thy. 2007.0327)

Schwarz-Lauer L, Chazenbalk GD, McLachlan SM, Ochi Y, Nagayama Y \& Rapoport B 2002 Evidence for a simplified view of autoantibody interactions with the thyrotropin receptor. Thyroid 12 115-120. (doi:10.1089/105072502753522347)

Tahara K, Ban T, Minegishi T \& Kohn LD 1991 Immunoglobulins from Graves' disease patients interact with different sites on TSH receptor/LH-CG receptor chimeras than either TSH or 
immunoglobulins from idiopathic myxedema patients. Biochemical and Biophysical Research Communications 179 70-77. (doi:10.1016/ 0006-291X(91)91335-A)

Tahara K, Ishikawa N, Yamamoto K, Hirai A, Ito K, Tamura Y, Yoshida S, Saito Y \& Kohn LD 1997 Epitopes for thyroid stimulating and thyroid blocking autoantibodies on the extracellular domain of the human thyrotropin receptor. Thyroid 7 867-877. (doi:10.1089/thy. 1997.7.867)
Thorpe IF \& Brooks CL 2007 Molecular evolution of affinity and flexibility in the immune system. PNAS 104 8821-8826. (doi:10. 1073 /pnas.0610064104)

Received in final form 8 November 2010

Accepted 1 December 2010

Made available online as an Accepted Preprint 19 January 2011 